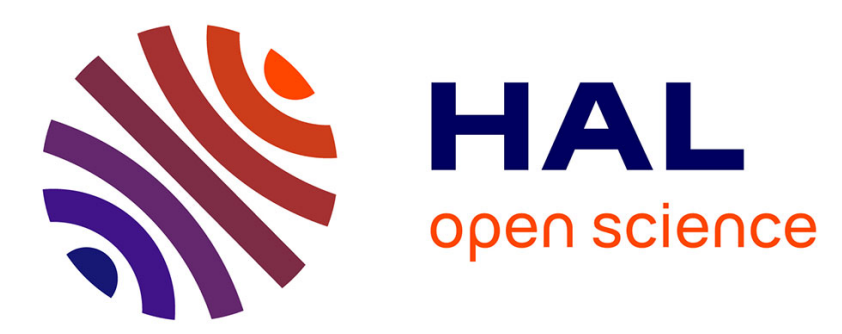

\title{
Time-domain computation of the response of composite layered anisotropic plates to a localized source
}

\author{
Éric Ducasse, Marc Deschamps
}

\section{To cite this version:}

Éric Ducasse, Marc Deschamps. Time-domain computation of the response of composite layered anisotropic plates to a localized source. Wave Motion, 2014, 18p. 10.1016/j.wavemoti.2014.08.003 . hal-01065967

\section{HAL Id: hal-01065967 \\ https://hal.science/hal-01065967}

Submitted on 18 Sep 2014

HAL is a multi-disciplinary open access archive for the deposit and dissemination of scientific research documents, whether they are published or not. The documents may come from teaching and research institutions in France or abroad, or from public or private research centers.
L'archive ouverte pluridisciplinaire HAL, est destinée au dépôt et à la diffusion de documents scientifiques de niveau recherche, publiés ou non, émanant des établissements d'enseignement et de recherche français ou étrangers, des laboratoires publics ou privés. 


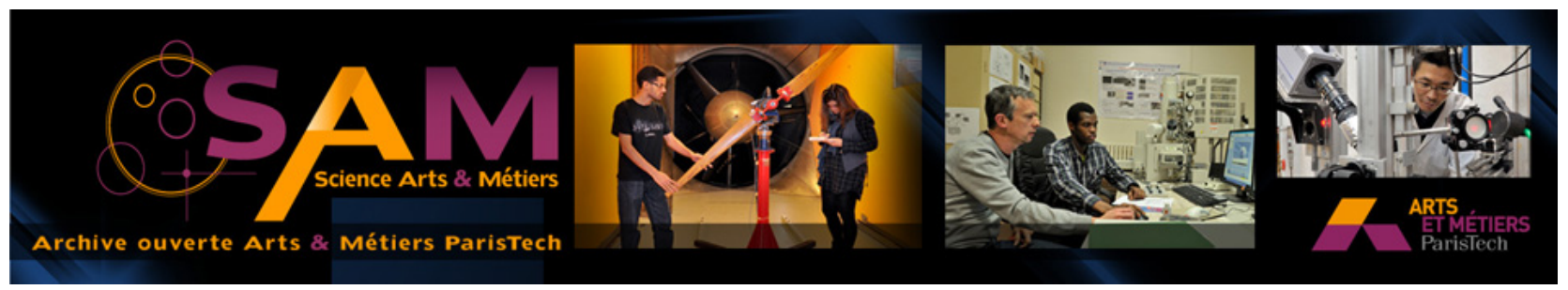

Science Arts \& Métiers (SAM)

is an open access repository that collects the work of Arts et Métiers ParisTech researchers and makes it freely available over the web where possible.

This is an author-deposited version published in: http://sam.ensam.eu Handle ID: .http://hdl.handle.net/10985/8562

\section{To cite this version :}

Éric DUCASSE, Marc DESCHAMPS - Time-domain computation of the response of composite layered anisotropic plates to a localized source - Wave Motion p.18p. - 2014 


\title{
Time-domain computation of the response of composite layered anisotropic plates to a localized source
}

\author{
Eric Ducasse ${ }^{1,2,3,4}$ e.ducasse@i2m.u-bordeaux1.fr \\ Marc Deschamps ${ }^{3,2}$ m.deschamps@i2m.u-bordeaux1.fr \\ 1 Arts et Metiers ParisTech, $I_{2} M-A P y$, UMR 5295, F-33400 Talence, France. \\ 2 Univ. Bordeaux, $I_{2} M-A P y$, UMR 5295, F-33400 Talence, France. \\ 3 CNRS, $I_{2} M-A P y$, UMR 5295, F-33400 Talence, France. \\ 4 Corresponding author. Tel. +33(0)540003138. Fax +33(0)540006964.
}

\begin{abstract}
This paper describes how a modal approach in the time-domain can be suitable for calculating the elastodynamic field in a layered plate. This elastodynamic field is generated by impulsive sources located in a small region of a composite plate consisting of anisotropic layers stuck together. The aim is to calculate the transient response of the elastic plate around the location of the sources, generally emitting $n$-cycle pulses. First, we apply a $2 \mathrm{D}$ Fourier transform to the wave equation with respect to the coordinates in the plate plane, and then, in the 2D spectrum domain, for any given wave-vector in the plate plane, solving a vibration problem with respect to time and position in the direction perpendicular to the plate. The solution is expressed as the sum of mode responses, each mode having a resonance frequency and a shape which depend on the wave-vector in the plate plane.
\end{abstract}

These calculations are different from those obtained by the usual method in the harmonic domain, where the modes are searched for a fixed frequency, such as Lamb waves, i.e. guided waves that propagate along the plate. In our case, the solution is given as a summation of plate resonances, i.e. a decomposition on the real eigenfrequencies, associated to Lamb waves with the same fixed wave-vector. This difference is of importance since only Lamb modes with real frequencies and real-valued wavenumbers in the plate plane are involved here, contrary to the usual harmonic methods, where these modes can be evanescent. This is of great interest as it can simplify the calculation of the generated field near the source.

Finally, we obtain a solution in the physical domain by performing an inverse 2D Fourier transform. After a detailed description of the method, results are shown for two typical plates. It is emphasized that the method is accurate for observation points located both above or below the source and reasonably far from it along the plate.

\section{Keywords}

time-domain; localized source; layered anisotropic plate; transient response. 


\section{Introduction}

The study of the diffraction of an ultrasonic source in an anisotropic multi-layered plate is of great interest in the field of Nondestructive Testing. From the point of view of propagation analysis, knowing the amplitude distribution of the elastodynamic field in different directions and studying ultrasonic wave propagation to the area of inspection are both important, for example, to choose the correct source for a specific problem under consideration. This is mainly true for inspections of composite materials, which are generally made of oriented fiber stacks. Their acoustic responses are complex and most of the time strongly anisotropic.

There are different harmonic methods to calculate the diffracted field, at any point and any time, in such plates (e.g., 1, 2]). The method most commonly used, without doubt, consists of applying a double Fourier transform to the initial partial differential equation in four variables, with respect to time and one direction $d$ in the plate plane. By doing so, we obtain a partial differential equation in a plane perpendicular to direction $d$, and consequently to the plate too, for which the angular frequency and the wavenumber in direction $d$ are parameters (see for example [3, 4]). The solution is then decomposed into guided modes, i.e. generalized Lamb waves, and the wavenumber in the direction along the plate perpendicular to direction $d$ is calculated from all the other spectrum variables. The guided wave can then be calculated by using a global matrix [5] for example, or an impedance matrix [6]. These methods are semi-analytic. Pure numerical methods exist like the SAFE method ([7], and e.g., [8]), which can be used to model very complex plane structures. When the modes are known, the residue theorem, or other techniques such as those based on the reciprocity theorem, can be applied to calculate mode amplitude (e.g., [3]). These field calculations are involved when developing hybrid methods, which consist of coupling mode theory with finite element methods [9, 8]. In essence, these approaches are well adapted to calculate the field for long distances of propagation along the plate, since the solution is given in terms of Lamb waves. In contrast, they are not so efficient for analyzing the field for sub-source locations, because a very large number of evanescent modes are involved in the field description.

There is an alternative technique, however, which is to do the calculation in the time-domain. Strangely, few studies can be found in the literature on this subject [10, 11]. In this case, a 2D Fourier transform is performed with respect to the coordinates in the plane of the layers (and of the interfaces) on the partial differential equation. In this $2 \mathrm{D}$ spectrum domain, i.e. at any fixed wave-vector $\mathbf{k}$, the solution is obtained by summation of plate resonances. A good introduction can be found in [10], showing that this method has been developed and exploited in geophysics. In this paper, we propose to develop this approach to study wave propagation in composite materials for Nondestructive Testing applications.

After a brief description of the basic equations (Section 2), using our notations, the solution is obtained in the time-domain (Section 3), analogously to what has been done by the so-called "Thin-Layer Method" [10], except that there is no discretization in the z-direction perpendicular to the plate. Indeed, in the Thin-Layer Method, "to solve the wave equation, [one] begins by dividing the physical domain in layers that are thin in the finite element sense [...]"[10]. Mode shapes are defined here analytically for the most general case as continuous functions of the $z$-coordinate, similarly to what is suggested for the isotropic case in [11. Nevertheless, at the last stage of the calculation, either semi-analytical or pure numerical techniques must be used to calculate the resonances of the plate. The finite difference method is used here for its efficiency but it is not necessary (see for example [5, 6] in the frequency-domain). In Section 4, the numerical aspects are presented and finally, in Section 5, different results are shown for two typical plates. It will be emphasized that the method is accurate for observation points located both under the source and reasonably far from it along the plate. 


\section{Equations in the physical domain}

Let us consider a multilayered medium consisting of a plate system with a number $m$ of perfect flat layers of normal $z$, stacked together. Each layer is an anisotropic solid, with a given thickness $h_{\beta}=z_{\beta}-z_{\beta-1}$, as illustrated in Fig. 1. The total thickness is expressed by $h$. Above and below this plate system are semi-infinite vacuum half-spaces. The plate is assumed to be infinite in the $x y$-plane, where the position is denoted by the vector $\mathbf{x}=(x, y)$.

This stratified medium is subjected to an external force density $f(\mathbf{x}, z, t)$ located between the planes $z=0$ and $z=h$ and in the cylinder of equation $|\mathbf{x}|<r_{s}$.

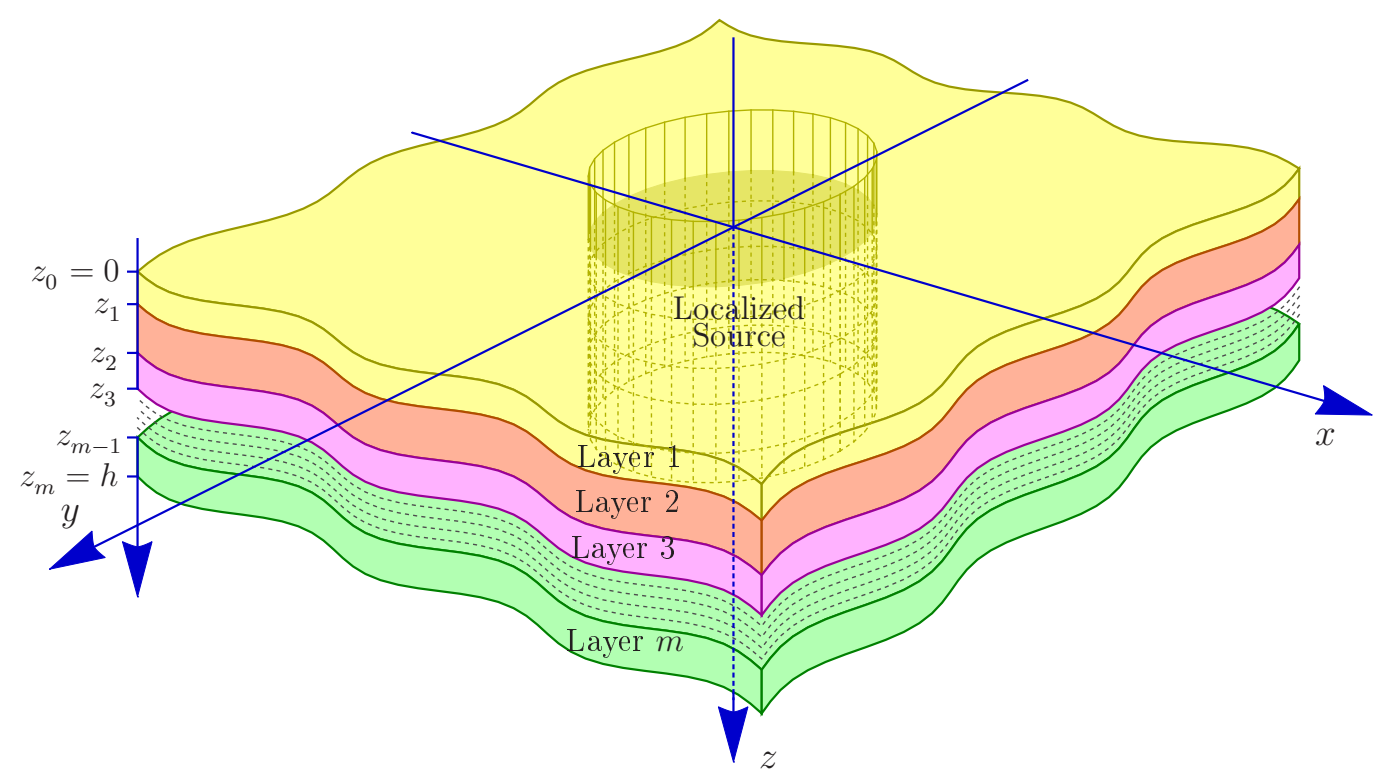

Figure 1: A multilayered infinite plate of thickness $h$ with $m$ layers.

\subsection{Equations in each layer}

In this section the equations of motion in each layer are presented. The development of these equations for an infinite homogeneous anisotropic solid has been covered in many textbooks, see for example [12, 13, 14. They are recalled here with condensed notations. At any time $t$ and any location $M(\mathbf{x}, z)$, the displacement field and the stress vector in the $\alpha$-direction are expressed by $\underline{\mathbf{u}}(\mathbf{x}, z, t)$ and $\underline{\boldsymbol{\sigma}}_{\alpha}(\mathbf{x}, z, t)(\alpha=x, y, z)$ respectively.

By applying Newton’s second law, equilibrium in layer $\sharp \beta$ requires:

$$
\rho_{\beta} \partial_{t}^{2} \underline{\mathbf{u}}(\mathbf{x}, z, t)=\partial_{\alpha} \underline{\boldsymbol{\sigma}}_{\alpha}(\mathbf{x}, z, t)+\underline{\mathbf{f}}(\mathbf{x}, z, t),
$$

where Einstein's summation convention over repeated indices is used.

Using the bilinear product $\stackrel{\beta}{\diamond}$, introduced in [15] and depending on the values of the elastic constants $c_{i j k m}^{[\beta]}$, Hooke's Law takes the form:

$$
\underline{\boldsymbol{\sigma}}_{\alpha}(\mathbf{x}, z, t)=\left(\mathbf{n}_{\alpha}{ }^{\diamond} \nabla\right) \underline{\mathbf{u}}(\mathbf{x}, z, t)
$$

where $\mathbf{n}_{\alpha}$ is the unit vector in the $\alpha$ direction, $\nabla=\partial_{\alpha} \mathbf{n}_{\alpha}$ is the gradient operator and $(\mathbf{a} \diamond \mathbf{b})$ is a three-by-three matrix such that $(\mathbf{a} \diamond \mathbf{b} \mathbf{b})_{i m}=c_{i j k m}^{[\beta]} a_{j} b_{k}$.

For simplicity, the index $\beta$ will be omitted below. It will be reintroduced only when necessary to avoid ambiguity. 
Combining equations (11) and (2) yields the following wave equation in each media:

$$
\rho \partial_{t}^{2} \underline{\mathbf{u}}(\mathbf{x}, z, t)-(\nabla \diamond \nabla) \underline{\mathbf{u}}(\mathbf{x}, z, t)=\underline{\mathbf{f}}(\mathbf{x}, z, t)
$$

with zero initial conditions.

\subsection{Boundary conditions and continuity at the interlayer surfaces}

Taking into account the free boundary conditions at both the upper and lower interfaces, the normal stresses in layer $\sharp 1$ at $z=0$ and in layer $\sharp m$ at $z=h$ are zero:

$$
(\mathbf{n} \diamond \nabla) \underline{\mathbf{u}}(\mathbf{x}, 0, t)=\mathbf{0} \text { and }(\mathbf{n} \diamond \nabla) \underline{\mathbf{u}}(\mathbf{x}, h, t)=\mathbf{0},
$$

with the notation $\mathbf{n}=\mathbf{n}_{z}$.

Completing the development of the equations, the continuity of the vector of displacements and of normal stresses at each interface between two consecutive layers, $\sharp \beta$ and $\sharp(\beta+1)$, must be satisfied. This implies:

$$
\underline{\mathbf{u}}\left(\mathbf{x}, z_{\beta}^{-}, t\right)=\underline{\mathbf{u}}\left(\mathbf{x}, z_{\beta}^{+}, t\right) \quad \text { and } \quad(\mathbf{n} \diamond \nabla) \underline{\mathbf{u}}\left(\mathbf{x}, z_{\beta}^{-}, t\right)=\left(\mathbf{n}^{\beta+1} \diamond \nabla\right) \underline{\mathbf{u}}\left(\mathbf{x}, z_{\beta}^{+}, t\right),
$$

where $z_{\beta}^{-}$indicates that the quantities are determined in layer $\sharp \beta$, while for $z_{\beta}^{+}$the same quantity is expressed in layer $\sharp(\beta+1)$.

It is of interest to note that each field exhibits a finite support at each time. As a matter of fact, since the source is localized, i.e. enclosed in a cylinder $|\mathbf{x}|<r_{s}$, and starts at $t=0$, the displacement field is necessarily zero outside of the cylinder $|\mathbf{x}|<\left(r_{s}+c_{\max } t\right)$, where $c_{\max }$ is the highest wave speed. This will be useful to calculate the 2D Fourier transform introduced in the next section.

\section{An infinity of one-dimensional vibrating systems}

The usual method used to solve Eqs. (3) to (5) is to apply a 3D Fourier transformation to them, with respect to $x, y$ and $t\left(e . g .,\left[5\right.\right.$, 16, 6]). The three space and time frequencies, denoted by $k_{x}, k_{y}$ and $\omega$, are independent variables. The first two are the components of the wave vector on the surface and the last one is the angular frequency. In each layer $\sharp \beta$ and for each partial mode $\sharp i$, the wave number $\kappa_{\beta, i}$ in the $z$-direction is then related to the three variables $k_{x}, k_{y}$ and $\omega$ by the Christoffel equation. Taking into account the boundary conditions, one spatial integral, for example those in $k_{x}$, is converted to an infinite summation of plate modes, which are obtained by searching all the guided waves with wave-numbers $k_{x, n}$, for given values of $k_{y}$ and $\omega$. The values of $k_{x, n}$ can be complex. Consequently, the guided waves can be exponentially attenuated in the $x$-direction. Similarly, the different values of the $i^{\text {th }}$ wavenumber $\kappa_{\beta, i, n}$ in the $z$-direction and in each layer can be complex according to the Christoffel equation.

In this paper, we present an alternative way to solve Eqs. (3) to (5) in the time-domain, extending what has been done by Kausel et al. in [10] and [11] to general anisotropy and continuous values of the vertical position $z$. To this end, the solution we are searching for in the plane of the plate is the superposition of real-valued sinusoidal fields, of wavelength $2 \pi k^{-1}$, where $k=|\mathbf{k}|$, as follows:

$$
\begin{aligned}
& \underbrace{\underline{\mathbf{u}}(\mathbf{x}, z, t)=\int_{\mathbb{R}^{2}} \cos (\mathbf{k} \cdot \mathbf{x}) \mathbf{u}_{\mathrm{c}}(\mathbf{k}, z, t)+\sin (\mathbf{k} \cdot \mathbf{x}) \mathbf{u}_{\mathrm{s}}(\mathbf{k}, z, t) \mathbb{d} \mathbf{k}}_{\mathbb{1}}
\end{aligned}
$$

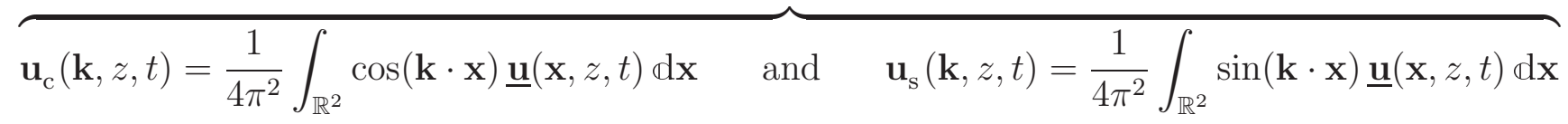


where $\mathbf{u}_{\mathrm{c}}(\mathbf{k}, z, t)$ and $\mathbf{u}_{\mathrm{s}}(\mathbf{k}, z, t)$ are independent real-valued quantities, which are even and odd functions respectively with respect to the wave-vector $\mathbf{k}$. As a consequence of these last properties, these two quantities can be grouped together in the following complex-valued field $\mathbf{U}(z, t)$ which is the usual Fourier transform of the displacement field $\underline{\mathbf{u}}(\mathbf{x}, z, t)$, such that:

$$
\mathbf{U}(z, t)=\mathbf{u}_{\mathrm{c}}(\mathbf{k}, z, t)+\dot{\mathrm{i}} \mathbf{u}_{\mathbf{s}}(\mathbf{k}, z, t)=\frac{1}{4 \pi^{2}} \int_{\mathbb{R}^{2}} \mathbb{e}^{\dot{\mathrm{i}} \mathbf{k} \cdot \mathbf{x}} \underline{\mathbf{u}}(\mathbf{x}, z, t) \mathbb{d} \mathbf{x} \Longleftrightarrow \underline{\mathbf{u}}(\mathbf{x}, z, t)=\int_{\mathbb{R}^{2}} \mathbb{e}^{-\dot{i} \mathbf{k} \cdot \mathbf{x}} \mathbf{U}(z, t) \mathbb{d} \mathbf{k},
$$

with $\mathbf{u}_{\mathrm{c}}(\mathbf{k}, z, t)=\mathcal{R} e[\mathbf{U}(z, t)]$ and $\mathbf{u}_{\mathrm{s}}(\mathbf{k}, z, t)=\operatorname{Im}[\mathbf{U}(z, t)]$. The dependence of this field with respect to the horizontal wave-vector $\mathbf{k}$ is omitted. Throughout this paper, when fields are denoted by upper case letters, they are expressed in the k-domain and the dependence with respect to the horizontal wave-vector is implicit.

\subsection{A one-dimensional oscillator for each horizontal wave-vector}

Finally the problem consists of finding the function $\mathbf{U}(z, t)$. Noting that the gradient operator $\nabla$ becomes $-\dot{\mathbf{i}} \mathbf{k}+\partial_{z} \mathbf{n}$ in the $\mathbf{k}$-domain, Eq. (3) is transformed into:

$$
\rho \partial_{t}^{2} \mathbf{U}(z, t)-(\mathbf{n} \diamond \mathbf{n}) \partial_{z^{2}} \mathbf{U}(z, t)+\dot{\mathbb{i}}[(\mathbf{n} \diamond \mathbf{k})+(\mathbf{k} \diamond \mathbf{n})] \partial_{z} \mathbf{U}(z, t)+(\mathbf{k} \diamond \mathbf{k}) \mathbf{U}(z, t)=\mathbf{F}(z, t),
$$

with zero initial conditions, where $\mathbf{F}(z, t)=\mathbf{f}_{\mathbf{c}}(\mathbf{k}, z, t)+\dot{\mathbf{i}} \mathbf{f}_{\mathbf{s}}(\mathbf{k}, z, t)$ is the Fourier transform of the force density $\underline{\mathbf{f}}(\mathbf{x}, z, t)$. This force field $\underline{\mathbf{f}}(\mathbf{x}, z, t)$ and the new complex function $\mathbf{F}(z, t)$ satisfy the same kind of relations introduced in Eqs. (6) and (7). Similarly, let us introduce the Fourier transform of the stress vector in the $\alpha$-direction, such that: $\Sigma_{\alpha}(z, t)=\boldsymbol{\sigma}_{\alpha \mathrm{c}}(\mathbf{k}, z, t)+\dot{\mathrm{i}} \boldsymbol{\sigma}_{\alpha \mathrm{s}}(\mathbf{k}, z, t)$.

Using this notation, the boundary conditions at both free interfaces are:

$$
\boldsymbol{\Sigma}_{z}(0, t)=(\mathbf{n} \diamond \mathbf{n}) \partial_{z} \mathbf{U}(0, t)-\dot{\mathrm{i}}(\mathbf{n} \diamond \mathbf{k}) \mathbf{U}(0, t)=\mathbf{0} \text { and } \boldsymbol{\Sigma}_{z}(h, t)=(\mathbf{n} \diamond \mathbf{n}) \partial_{z} \mathbf{U}(h, t)-\dot{\mathrm{i}}(\mathbf{n} \diamond \mathbf{k}) \mathbf{U}(h, t)=\mathbf{0},
$$

while the equations of continuity at each interface between two layers are:

$$
\begin{aligned}
\mathbf{U}\left(z_{\beta}^{-}, t\right) & =\mathbf{U}\left(z_{\beta}^{+}, t\right) \text { and } \\
(\mathbf{n} \diamond \mathbf{n}) \partial_{z} \mathbf{U}\left(z_{\beta}^{-}, t\right)-\dot{\mathrm{i}}(\mathbf{n} \diamond \mathbf{k}) \mathbf{U}\left(z_{\beta}^{-}, t\right) & =\left(\mathbf{n}^{\beta+1} \diamond \mathbf{n}\right) \partial_{z} \mathbf{U}\left(z_{\beta}^{+}, t\right)-\dot{\mathbb{i}}\left(\mathbf{n}^{\beta+1} \diamond^{\beta}\right) \mathbf{U}\left(z_{\beta}^{+}, t\right) .
\end{aligned}
$$

The initial problem is then replaced by equation (8) of motion in each layer, which is coupled with the boundary conditions (9), and the different equations (10) of continuity. Thus, for a given vector $\mathbf{k}$, we obtain a partial differential equation system with respect to position $z$ and time $t$. In fact, we are searching for the displacement response $\mathbf{U}$ of a one-dimensional vibrating system (similar to a string or a beam) to the excitation $\mathbf{F}$. This vibrating system is constituted by $m$ media, as shown in Fig. 2 ,

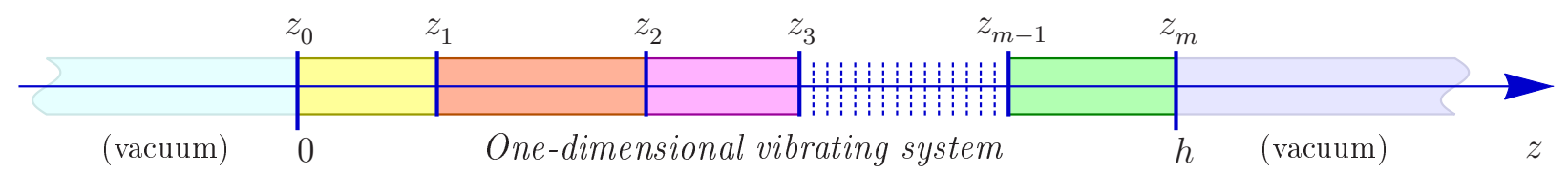

Figure 2: A string-like one-dimensional vibrating system of length $h$, including $m$ media. 


\subsection{Decomposition of the response in the modal basis}

As is usually the case in structural dynamics (e.g., [17]), equations (8), (9) and (10) lead to the following weak formulation:

$$
\forall \boldsymbol{\Phi},\left\langle\boldsymbol{\Phi}, \partial_{t}^{2} \mathbf{U}(\bullet, t)\right\rangle+\mathbb{A}(\mathbf{\Phi}, \mathbf{U}(\bullet, t))=\left\langle\boldsymbol{\Phi}, \rho^{-1} \mathbf{F}(\bullet, t)\right\rangle,
$$

where the scalar product of two displacement fields $\boldsymbol{\Phi}$ and $\boldsymbol{\Psi}$ is defined by:

$$
\langle\boldsymbol{\Phi}, \boldsymbol{\Psi}\rangle=\int_{0}^{h}\left[\mathbf{\Phi}(z)^{+} \mathbf{\Psi}(z)\right] \rho(z) \mathbb{d} z,
$$

the superscript + denoting the transposition combined with the complex conjugation, and where the positive hermitian form $\mathbb{A}$ is expressed as follows:

$$
\mathbb{A}(\boldsymbol{\Phi}, \boldsymbol{\Psi})=\int_{0}^{h} \boldsymbol{\Phi}^{\prime}(z)^{+}\left[(\mathbf{n} \diamond \mathbf{n}) \boldsymbol{\Psi}^{\prime}(z)-\dot{\mathrm{i}}(\mathbf{n} \diamond \mathbf{k}) \boldsymbol{\Psi}(z)\right]+\dot{\mathrm{i}} \boldsymbol{\Phi}(z)^{+}\left[(\mathbf{k} \diamond \mathbf{n}) \boldsymbol{\Psi}^{\prime}(z)-\dot{\mathrm{i}}(\mathbf{k} \diamond \mathbf{k}) \boldsymbol{\Psi}(z)\right] \mathbb{d} z .
$$

The solution $\mathbf{U}$ of Eq. (11) is expressed in the orthonormal basis of the eigenmodes $\left(\omega_{n}, \boldsymbol{\Phi}_{n}\right)$ of the onedimensional oscillator as follows (see Appendix A for more details):

$$
\mathbf{U}(z, t)=\sum_{n=1}^{+\infty} u_{n}(t) \Phi_{n}(z)
$$

$u_{n}(t)$ denoting the magnitude of the mode $\sharp n$.

By replacing $\Phi$ by $\Phi_{n}$ in Eq. (11) for each mode $\sharp n$, successively, and by using both the orthogonality property (A.9) and Eq. (A.11), we obtain the following independent ordinary differential equations in the time-domain governing each magnitude $u_{n}(t)$ :

$$
\forall n \in \mathbb{N}^{*}, u_{n}^{\prime \prime}(t)+\omega_{n}^{2} u_{n}(t)=\int_{0}^{h} \boldsymbol{\Phi}_{n}(z)^{+} \mathbf{F}(z, t) \mathbb{d} z=f_{n}(t),
$$

with zero initial conditions. The $f_{n}(t)$ coefficient results from the projection of the source-term $\mathbf{F}$ divided by the density $\rho$ onto the mode shape $\sharp n$, i.e. $\rho(z)^{-1} \mathbf{F}(z, t)=\sum_{n=1}^{+\infty} f_{n}(t) \boldsymbol{\Phi}_{n}(z)$.

Thus, the exact solution of Eq. (13) is written as follows:

$$
u_{n}=\left[\mathfrak{h}(\bullet) \frac{\sin \left(\omega_{n} \bullet\right)}{\omega_{n}}\right] * f_{n},
$$

where $\mathrm{h}$ is the Heaviside unitstep function (omitted in [10] and [11]) and $*$ denotes the continuous-time convolution.

\subsection{Synthesis of the displacement field in the physical domain}

Finally the response $\mathbf{U}(z, t)$, at any fixed time $t$ and at any vertical position $z$ of observation, was obtained from Eqs. (12) and (14) for all wave-vectors k. An inverse 2D Fourier transform by Eq. (7) yields a snapshot of the displacement field at time $t$ and at depth $z$. The direct and inverse 2D Fourier transform have to be done numerically, as shown in the next section. 


\section{Computation}

Once a rectangular domain of observation and discretization steps have been chosen, the computation is performed in two successive stages: firstly, for a given layered plate, the different modes are calculated and stored. The mode computation has to be done once and for all. Secondly, the time-domain response of the plate to a given solicitation is calculated.

\subsection{Discretization and periodization in the $\mathrm{x}$ - and k-domains}

As noted below, the displacement field at any time $t$ is zero outside of a cylinder of radius $\left(r_{s}+c_{\max } t\right)$. Consequently, two values $x_{\max }$ and $y_{\max }$ can be chosen such that $\underline{\mathbf{u}}(\mathbf{x}, z, t)=\mathbf{0}$ if the horizontal position $\mathbf{x}$ is outside of the rectangle $\left[-x_{\max }, x_{\max }\right] \times\left[-y_{\max }, y_{\max }\right]$ and if time $t$ is less than $t_{\max }$.

According to the Shannon Sampling Theorem (e.g., [18]) applied to a 2D space, if the source is assumed to contain only spatial frequencies less than $k_{x}^{\max }$ and $k_{y}^{\max }$ in the $x$ - and $y$-directions respectively, the $2 \mathrm{D} \mathbf{x}$ (physical space) and k-domains (horizontal wave-vectors) can be discretized.

Thus, a $n_{x}$-by- $n_{y}$ grid is defined on each domain. The discretization steps are $\delta x$ and $\delta y$ in the $\mathbf{x}$-domain, $\delta k_{x}$ and $\delta k_{y}$ in the k-domain. They satisfy the following relations:

$$
\delta x=\frac{2 x_{\max }}{n_{x}}=\frac{\pi}{k_{x}^{\max }} ; \delta y=\frac{2 y_{\max }}{n_{y}}=\frac{\pi}{k_{y}^{\max }} ; \quad \delta k_{x}=\frac{2 k_{x}^{\max }}{n_{x}}=\frac{\pi}{x_{\max }} ; \delta k_{y}=\frac{2 k_{y}^{\max }}{n_{y}}=\frac{\pi}{y_{\max }} .
$$

In fact, our goal is to compute the real values of the displacement field on the grid in the $\mathbf{x}$-domain. These values are obtained by applying Fast Fourier Transforms [19] to the displacement field computed in the k-domain by Eqs. (12) and (14). Due to the symmetry properties of Eq. (6), the latter computation must be made on set $\mathcal{G}$ only, as this set almost corresponds to the half of the grid in the k-domain:

$$
\mathcal{G}=\left\{\left(l_{x} \delta k_{x}, l_{y} \delta k_{y}\right) ; l_{y}=0 \text { and } 0 \leqslant l_{x} \leqslant n_{x} / 2, \text { or } 1 \leqslant l_{y} \leqslant n_{y} / 2 \text { and } 1-n_{x} / 2 \leqslant l_{x} \leqslant n_{x} / 2\right\}
$$

\subsection{Modes computation}

We search for numerical values for each eigenmode $(\omega, \boldsymbol{\Phi})$ that will approximately satisfy the following equations. Firstly, in each layer:

$$
(\mathbf{n} \diamond \mathbf{n}) \Phi^{\prime \prime}(z)-\dot{\mathbb{i}}[(\mathbf{n} \diamond \mathbf{k})+(\mathbf{k} \diamond \mathbf{n})] \boldsymbol{\Phi}^{\prime}(z)+\left[\rho \omega^{2} \mathbb{I}_{3}-(\mathbf{k} \diamond \mathbf{k})\right] \boldsymbol{\Phi}(z)=\mathbf{0} .
$$

Secondly:

$$
(\mathbf{n} \diamond \mathbf{n}) \Phi^{\prime}(0)-\dot{\mathbb{i}}(\mathbf{n} \diamond \mathbf{k}) \boldsymbol{\Phi}(0)=\mathbf{0} ;(\mathbf{n} \diamond \mathbf{n}) \Phi^{\prime}(h)-\dot{\mathbb{i}}(\mathbf{n} \diamond \mathbf{k}) \boldsymbol{\Phi}(h)=\mathbf{0} \text { (free walls). }
$$

And lastly, the continuity at each interlayer $\sharp \beta$ is expressed by:

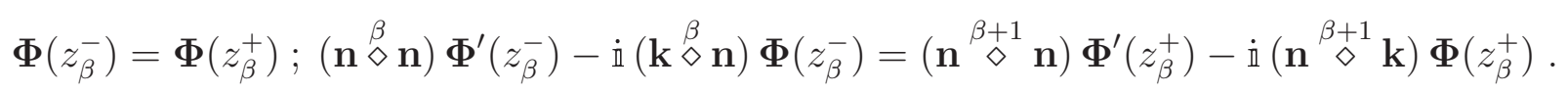

Because the second-order ordinary differential equation (17,a) on each layer is linear and parameterized by the wave-vector $\mathbf{k}$ and the $\left(\rho \omega^{2}\right)$ coefficient, its solution space is six-dimensional and can be determined analytically. For each of the $m$ layers, the solution $\boldsymbol{\Phi}(z)$ is then characterized by six coefficients associated to six exponential solutions, each one defined by a wave-number in the $z$-direction and a polarization vector. Consequently, the problem is to find the $6 m$ coefficients that satisfy the 6 equations (17, b) and the $6(m-1)$ equations (17, c). Thus, 
we have to find non-zero solutions of a $6 m$-by- $6 m$ linear system, i.e. to determine the frequencies $\omega$ such that the determinant of a $6 m$-by- $6 m$ matrix is zero. In fact, we obtain a Pochhammer-like dispersion equation which is strongly non-linear and can be difficult to solve numerically.

An alternative approach is to discretize the interval $[0, h]$ and replace the first-order and second-order derivatives with respect to $z$ in Eq. (17) by finite differences. Note that a finite element method could also be used, as is usually done in the frequency-domain (SAFE method [7, 9, 8]). Thus, the present approach could be called the "Time-Domain Semi-Analytical Finite Difference" (TDSAFD) method. After discretization, Eq. (17) becomes an ordinary eigenvalue problem $\mathbb{M} \boldsymbol{\varphi}=\omega^{2} \boldsymbol{\varphi}$, where $\mathrm{M}$ is a sparse square matrix.

After computation, each mode is recorded by storing its eigenfrequency and the $6 m$ pairs (wave-number in the $z$ direction, polarization vector multiplied by the amplitude coefficient). In practice, a maximum frequency $\omega_{\max }$ has to be considered such that each excitation contains frequencies lower than $\omega_{\max }$. The number of modes obtained for the two numerical examples given below are drawn in Fig. 3 with respect to the horizontal wavevector $\mathbf{k}$. One can see that the number of modes decreases and tends to zero when the wave-number (the norm of the wave-vector) increases. It is important to notice that the modes to take into account, i.e. of eigenfrequencies less than the maximum frequency of the excitation signal, are necessarily in a finite number, contrarily to standard frequency-domain methods. Indeed, in this latter case, an infinity of evanescent modes has to be neglected. Fig. 3(a) shows that only 8 modes are needed to calculate the response of a monolayer carbon epoxy plate of $3.6 \mathrm{~mm}$ thickness to a source of $500 \mathrm{kHz}$ maximum frequency. As the maximum excitation frequency increases, the number of required modes increases. For example, approximately 60 modes are required for a maximum frequency of $5 \mathrm{MHz}$. Empirically, we also obtained the best estimate of the eigenfrequencies (up to eight significant digits) by eighth-order and tenth-order finite difference schemes and a sufficient number of subintervals of discretization, which obviously must increase as the maximum excitation frequency increases. The comparison was made possible by using an additional (costly) numerical method on the non-linear dispersion equation resulting from Eq. (17) to find each "exact" eigenfrequency (double precision calculation) in the close vicinity of each computed eigenfrequency.

(a)

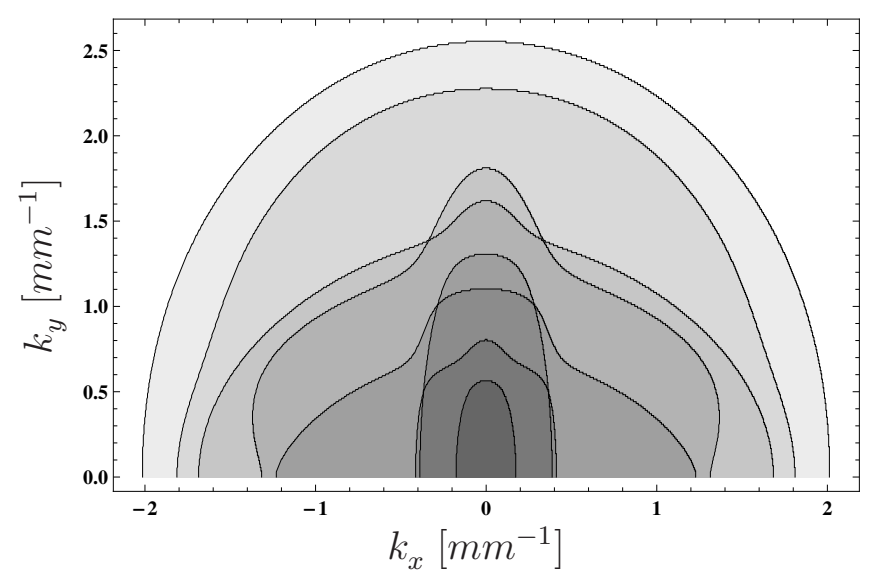

From 0 (white) to 8 modes (darkest zone)

Frequencies lower than $500 \mathrm{kHz}$

$k_{x}^{\max }=2.10 \mathrm{~mm}^{-1}, n_{x}=1024, \delta x \approx 1.496 \mathrm{~mm}$

$k_{y}^{\max }=2.60 \mathrm{~mm}^{-1}, n_{y}=512, \delta y \approx 1.208 \mathrm{~mm}$ (b)

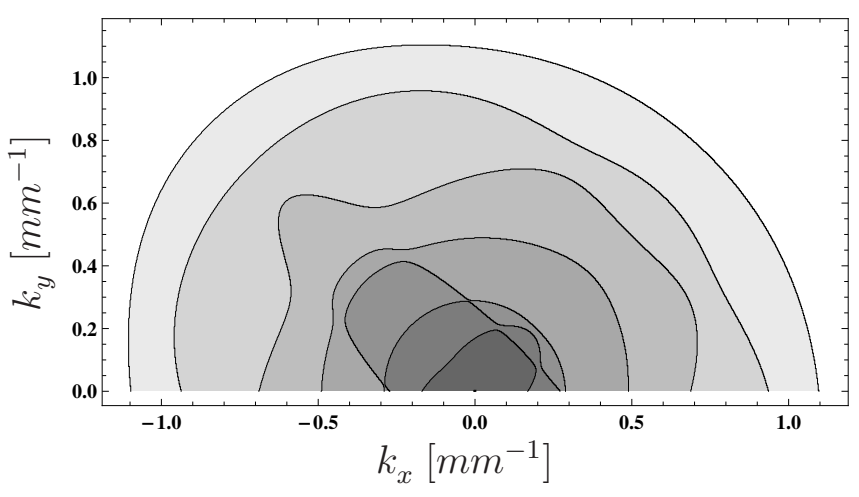

From 0 (white) to 7 modes (darkest zone)

Frequencies lower than $250 \mathrm{kHz}$

$k_{x}^{\max }=1.142 \mathrm{~mm}^{-1}, n_{x}=1024, \delta x \approx 2.75 \mathrm{~mm}$

$k_{y}^{\max }=1.142 \mathrm{~mm}^{-1}, n_{y}=1024, \delta y \approx 2.75 \mathrm{~mm}$

Figure 3: Number of calculated modes with respect to the horizontal wave-vector: two examples.

(a) A monolayer carbon epoxy plate of thickness $3.6 \mathrm{~mm}$.

(b) A three-layer carbon epoxy/glue/steel plate of thicknesses $3.6 \mathrm{~mm} / 0.3 \mathrm{~mm} / 1.0 \mathrm{~mm}$. 


\subsection{Response building}

We start from the knowledge of the external force density $f(\mathbf{x}, z, t)$. The first step consists of calculating its 2D Fourier transform $\mathbf{F}(z, t)$ for each horizontal wave-vector $\mathbf{k}$ of set $\mathcal{G}$ [see Eq. (16)]. Generally, $\mathbf{F}(z, t)$ will be computed by FFT but for basic shapes like the Gaussian point-sources used for the numerical examples below, it can be expressed analytically. In the second step, the source term $\mathbf{F}(z, t)$ is projected on the modal basis, for each $\mathbf{k}$ in $\mathcal{G}$. This projection leads to the $f_{n}$ functions defined in Eq. (13). As in the first step, these functions can be either symbolic or numerical expressions.

In the examples below, we consider the particular case of a surface force exerted on the upper side of the plate at $z=0$. Thus, the volume force density becomes:

$$
\mathbf{x} \text {-domain : } \mathbf{f}(\mathbf{x}, z, t)=\delta(z) s(t) \mathbf{g}(\mathbf{x}) \Longleftrightarrow \mathbf{k} \text {-domain : } \mathbf{F}(z, t)=\delta(z) s(t) \mathbf{G},
$$

where $\delta$ denotes the Dirac distribution, $s(t)$ is a dimensionless signal, $\mathbf{g}(\mathbf{x})$ is the surface force density, and the $\mathbf{G}$ vector its $2 \mathrm{D}$ Fourier transform. In this case, the projection of the source onto the mode $\sharp n$ becomes: $f_{n}(t)=\left[\boldsymbol{\Phi}_{n}(0)^{+} \mathbf{G}\right] s(t)$.

The third step consists of calculating the convolution (14) to obtain the amplitude $u_{n}(t)$ of the mode $\sharp n$. This point may need more study in the general case but here we consider only Gaussian pulses and $n$-cycle pulses, for which the convolution is symbolic, as shown in Appendix B.

\section{$5 \quad$ Numerical results}

This section reports some numerical results based on our numerical schemes. Two typical plates are analyzed. The first plate is made of a single layer of a unidirectional fiber composite. The second is a structure made of a unidirectional fiber composite plate and a steel plate stuck together.

\subsection{Monolayer carbon epoxy plate}

Within the frequency range of calculations, such a composite material can be considered as an orthotropic homogeneous medium for which the equivalent density is $\rho=1560 \mathrm{~kg} \cdot \mathrm{m}^{-3}$ and the homogenized stiffnesses are: $c_{11}=86.60, c_{22}=13.50, c_{33}=14.00, c_{44}=2.72, c_{55}=4.06, c_{66}=4.70, c_{12}=9.00, c_{13}=6.40, c_{23}=6.80$ [GPa]. The crystallographic directions $\sharp 1, \sharp 2$ and $\sharp 3$ correspond to the $x$-, $y$ - and $z$-axis, respectively. The direction of fibers coincides with the $x$-axis. This material has been chosen because some experiments have already been carried out on it [20, Table 2.3], some of which will be discussed later. The plate thickness is $3.6 \mathrm{~mm}$. The source is a 5 -cycle pulse of frequency $150 \mathrm{kHz}$ normally exerted on the upper side of the plate (in the $z$-direction) as an axisymmetric Gaussian force density $15 \mathrm{~mm}$ wide, i.e. the product of two Gaussian functions with respect to $x$ and $y$, respectively, with the same width of $15 \mathrm{~mm}$ [see Eq. (B.1), where "duration" is replaced by "width", and Eq. (B.3) for exact definitions].

First of all, let us determine the modes that can be generated in the inspection conditions. In the frequency range of the emitter and for the carbon epoxy sample, there are only four possible propagating modes. The three fundamental modes $A_{0}$ (flexural wave), $S H_{0}$ (horizontal shear wave) and $S_{0}$ (compressional wave), and the first anti-symmetric mode, generally named $A_{1}$ for the isotropic case. Even though this classification is improper for anisotropic plates, we will use it for simplicity.

Compared to the group velocity of bulk waves, where the dispersion is only angular, the dispersion of Lamb modes is a more complex phenomenon since it is both angular and frequential. The group velocity vector, which defines the wave-front, is normal to the phase slowness surface and takes into account the variation in the phase 
velocity versus the frequency. The complexity of the group velocity of Lamb modes propagating in anisotropic media has been emphasized in Figure 4, where the phase and group velocities, in the interface plane, are plotted in polar coordinate for the frequency range of the emitter pulse. In this frequency range, the dependence of these two velocities on the frequency and on the angle of observation is clearly visible. The extent of this dependence depends on the mode. In this figure, for both velocities, the curves associated to the central frequency are plotted in a solid line. Note that for the $A_{1}$ mode, this specific curve is not plotted since its associated cut-off frequency is greater than the central frequency.

(a)

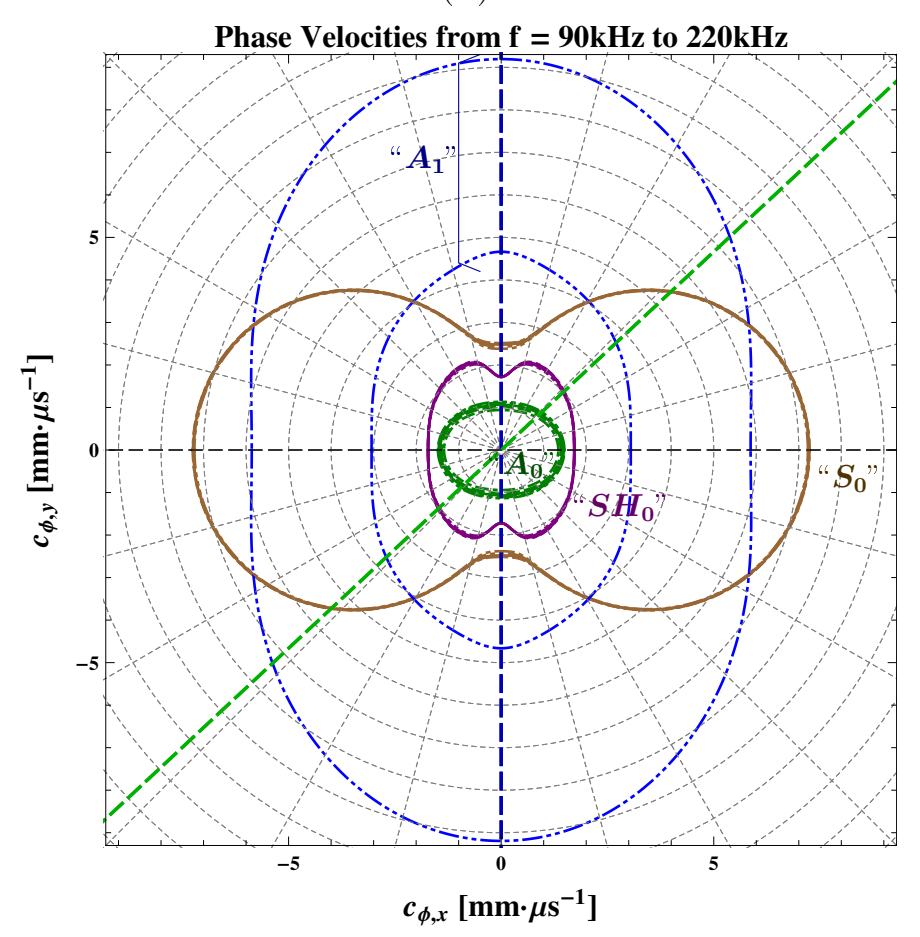

(b)

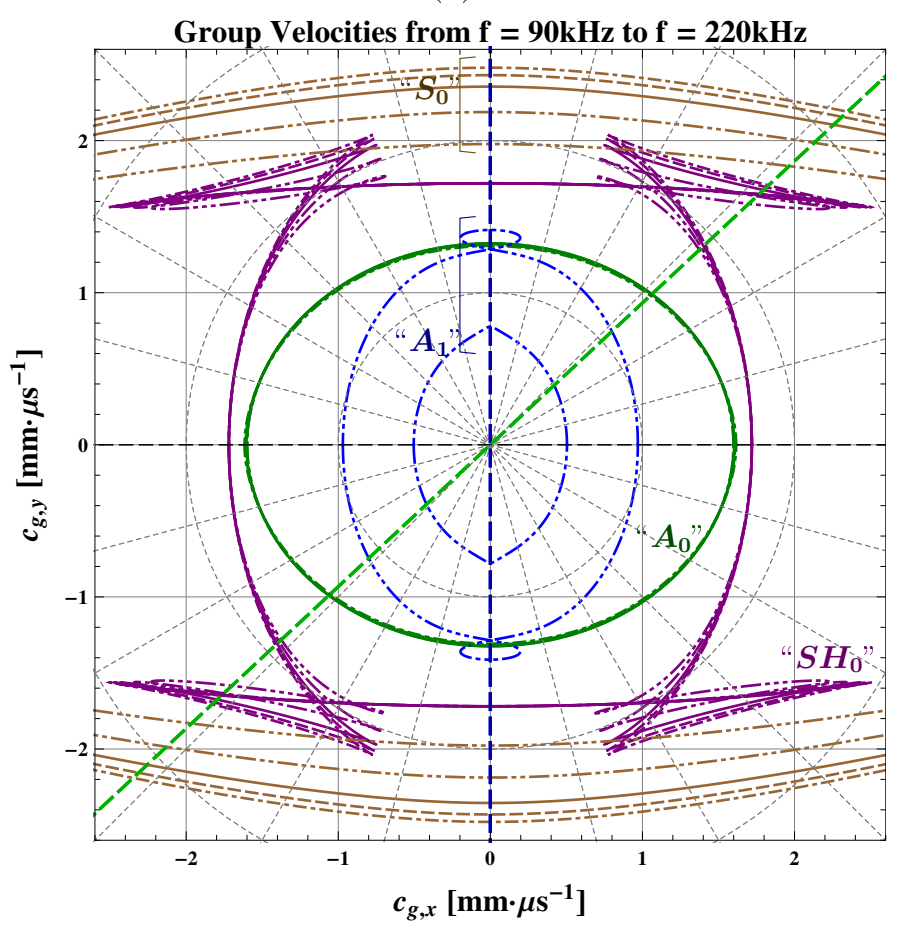

Figure 4: (a) Phase and (b) group velocities of a carbon epoxy plate of thickness $3.6 \mathrm{~mm}$ at the central frequency $150 \mathrm{kHz}$ (solid line), lower frequencies $130 \mathrm{kHz}$ (dashed), $110 \mathrm{kHz}$ (dot-dashed) and $90 \mathrm{kHz}(-\cdot \cdot)$, and higher frequencies $170 \mathrm{kHz}$ (long-dashed), $190 \mathrm{kHz}$ (dot-long-dashed), $200 \mathrm{kHz}$ (—..), $210 \mathrm{kHz}$ (一 - - ) and $220 \mathrm{kHz}$ (dotted).

The wave packets, associated to the four modes, can be observed on the surface field, as shown in Fig. 5 . Fig. [5(a)-(c) represents, respectively, the angular, radial and vertical displacements. The wave-fronts associated to the ray theory, i.e. the energy velocity curves, are also plotted for the central frequency. The three fastest fronts correspond to the three fundamental modes $A_{0}, S H_{0}$ and $S_{0}$. The good agreement between the field calculation and the ray theory is noticeable. This is mainly remarkable for the cusp of the $S H_{0}$ wave-front, which is, in addition, in agreement with some experimental measurements that have been done with the same sample [20]. Moreover, although the central frequency is below the first cut-off frequency, the energy after this limit frequency is large enough to generate the $A_{1}$ mode. This is a very slow wave packet which reveals a very strong dispersive mode. Indeed, both phase and group velocities of this $A_{1}$ mode strongly depend on frequency, as shown in Fig. 4. As frequency increases, phase velocity decreases (see Fig. 4(a)), i.e. wavelength decreases, while group velocity increases (see Fig. 4(b)). In accordance with this property, it can be observed for the $A_{1}$ mode in Fig. 5 that the further away you get from the source, the lower the half-wavelength is.

Specific analysis for fixed angles of observation are of great interest. To carry out these complementary investigations, the two typical angles of $\theta=90^{\circ}$ and $\theta=43^{\circ}$ have been chosen because they correspond to two cases with and without cusp phenomena. These angles are identified by blue and green dashed lines in Figs. 4 and 5 .

First, the displacement field over the thickness is calculated. For the two cross-sections associated to the angles $\theta=90^{\circ}$ and $\theta=43^{\circ}$, Figs. 6 and 8 show the three components of the displacement vectors in the corresponding cross-sections at the fixed time $t=100 \mu s$ (the same time as for Fig. 可). The fixed observation time is long enough 
(a) Radial Displacement

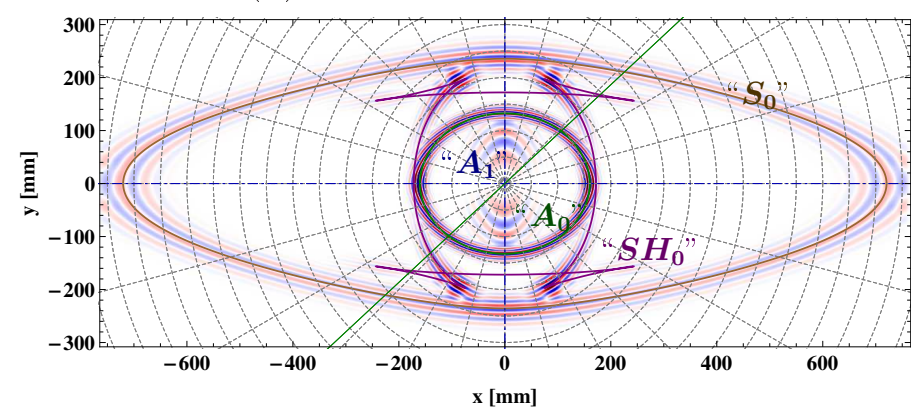

(c) Vertical Displacement

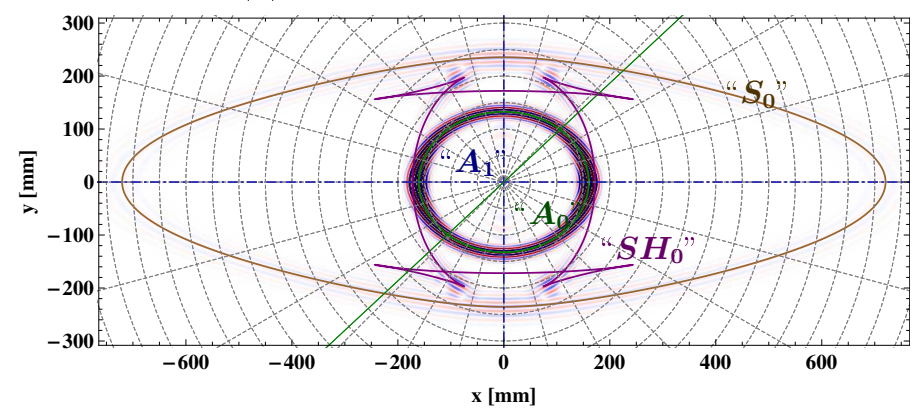

(b) Angular Displacement

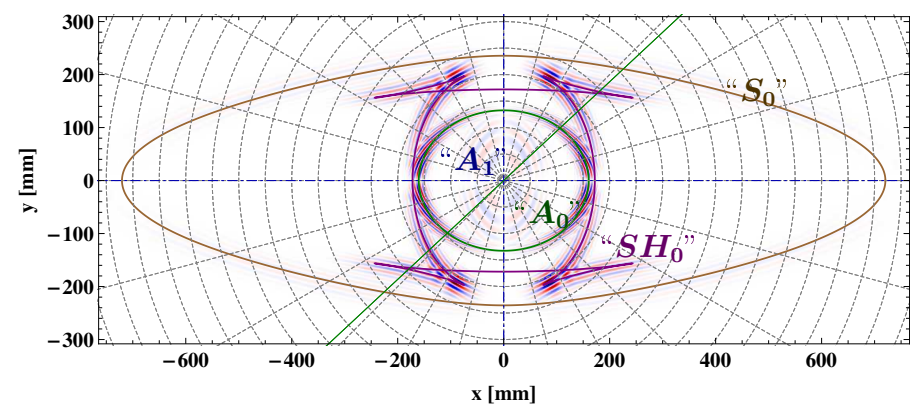

Figure 5: The displacements at $z=0$ and $t=100 \mu \mathrm{s}$ in a carbon epoxy plate of thickness $3.6 \mathrm{~mm}$ excited by a 5 -cycle pulse of frequency $150 \mathrm{kHz}$ at $z=0$.

for the Lamb waves to become well established and to separate their wave packets one from another. Second, the oscillations of the top surface, i.e. at $z=0$, are shown for various times, in Figs. 7 and 9 , for the two chosen observation directions. In these two figures, different wave-fronts are identified by their group velocities $c_{g}$, which are in good agreement with the group velocities at the central frequency drawn in Figs. 4(b) and 5. As a general observation, when the modes are generated, note the good correspondence between the energy velocity and the maximum of oscillations. Let us now focus our interest on particular observations involving the two angles.

For the angle $\theta=90^{\circ}$, in Figs. 6 and 7, only three Lamb waves are clearly visible on the displacement field vector over the cross-section and on the top surface oscillations, as the $S H_{0}$ mode is not generated in this direction by this source (see also Fig. 5).

For the generated modes, the field shape over the cross-section is, at some particular distances from the source, close to the mode shape associated to one of these modes. In fact, the symmetric and the antisymmetric behavior, which depends on the wave field component, is clearly well defined on the mode shape. It is also discernible for the very dispersive mode $A_{1}$. In this case, it is noteworthy that the pseudo-periodicity observed for the displacement at the top surface still exists over the section, while respecting the symmetry and antisymmetry of the mode shapes. For all these main contributions to the field, because the propagation direction is following a principal direction, the mode shapes correspond to Lamb waves for which the phase velocities are also oriented in this direction. From a numerical point of view, calculation of the wave fields has been deliberately left erroneous. These wave fields are visible at the top right of Fig. 7, for observation times $t$ greater than $t_{\max } \approx 120 \mu s$. These artifacts come from the choice of space sampling when using the FFT algorithm. According to the Shannon theorem, they correspond to waves produced by the periodized source $\left(\delta k_{x}^{-1}\right.$ in the $x$-direction and $\delta k_{y}^{-1}$ in the $y$-direction, see 4.1 . In this particular case, the observed wave is produced by the source located at point $\left(0, \delta k_{y}^{-1}\right)$. The size of the discretized rectangular domain depends, among other things, on the maximum horizontal velocity (in the $x y$-plane) as well as on the maximum time of observation $t_{\max }$. Thus, the longer the observation time, the greater the discretized rectangular domain must be. A simple extension of this domain, which requires smaller discretization steps in the wave-vector domain, would have avoided this problem. The cost would have been a significant increase in calculation time. Consequently, far from the source and for long 
observation times, standard frequency-domain methods will be more efficient than the present time-domain method. Indeed, in this case, only a few propagating Lamb modes are generally sufficient to describe the ultrasonic field. Thus, the closer to the source the observation domain is, in time and space, more efficient the present method is.

(a) Radial displacement

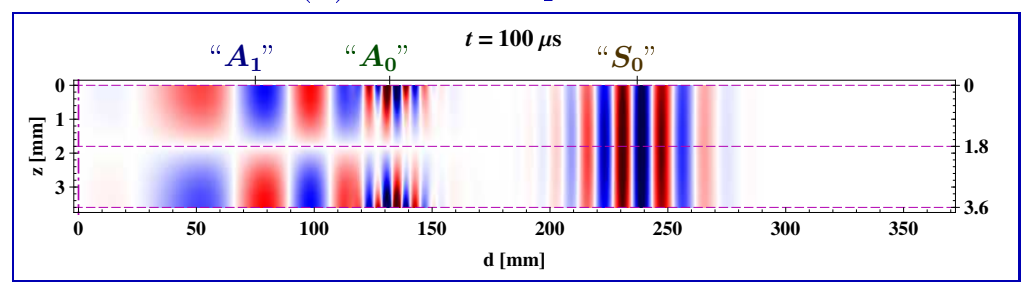

(b) Angular displacement

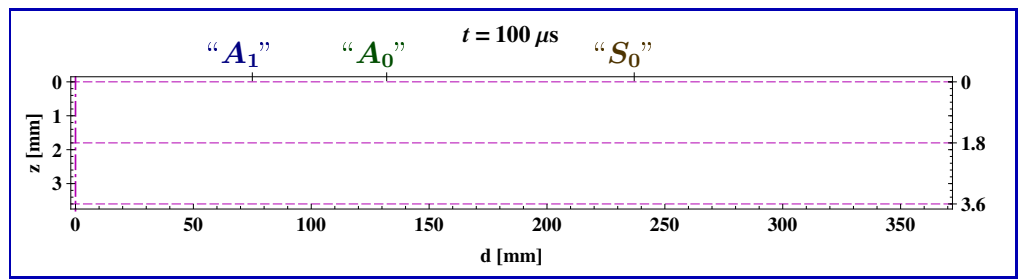

(c) Vertical displacement

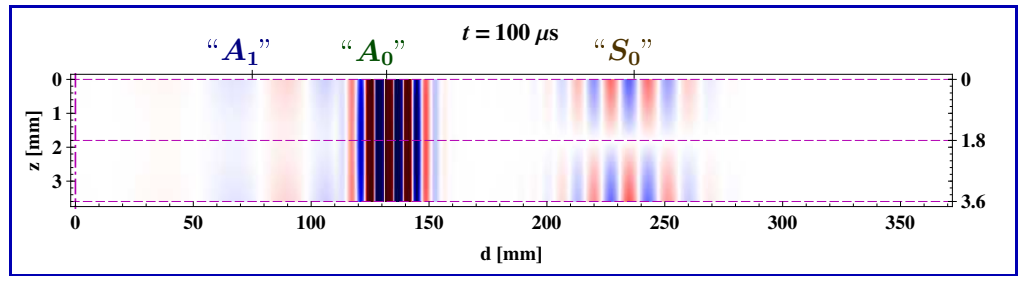

Figure 6: The displacements on the section $\theta=90^{\circ}$ at $t=100 \mu \mathrm{s}$ in a carbon epoxy plate of thickness $3.6 \mathrm{~mm}$ excited by a 5 -cycle pulse of frequency $150 \mathrm{kHz}$ at $z=0 . d$ is the distance from the source.

For the angle $\theta=43^{\circ}$, the presence of the cusp, as is well known, leads to multiple ray contributions of a single Lamb mode. For the "SH " mode, these different contributions are clearly observed in the field on the top surface. It has been observed in the wave field, as has been well described in the literature, see for example [20], and measured experimentally [21]. In this paper, in addition to the method used, what is interesting in the results is the analysis of the displacements in such a cross-section, i.e. with observable cusp phenomenon. A close study of the mode shapes, identified in Fig. 8, reveals that each of them is associated to the mode shape of the Lamb wave associated to the ray propagating in the direction of the cross-section. In addition, the wavelength of each identified oscillation is directly connected to the projection of the phase velocity in the direction of the cross-section.

To examine how the calculations are possible near to the source, i.e. in the close field, let us analyze in Fig. 10 the radial displacement $u_{r}$ in the cross-section $x=0$ during the first microseconds. In this figure, we plot the shape of the field following the $z$-direction against the distance $d=y$ at different times. Because the excitation source starts at one negative time ( $c f$. Appendix B) the analysis is done from $t=-15 \mu s$ up to $t=13.5 \mu s$. For the shortest excitation times, i.e. for negative times, the acoustic field is, of course, concentrated in the vicinity of the source. For such observation points, a lot of waves interact. However, the field computation is done correctly. As the time increases, it is interesting to observe the modes building. In fact, for the longest times, the $S_{0}$ mode (compressional wave) is well established and the mode shape of the $A_{0}$ mode (flexural wave) starts to emerge. 


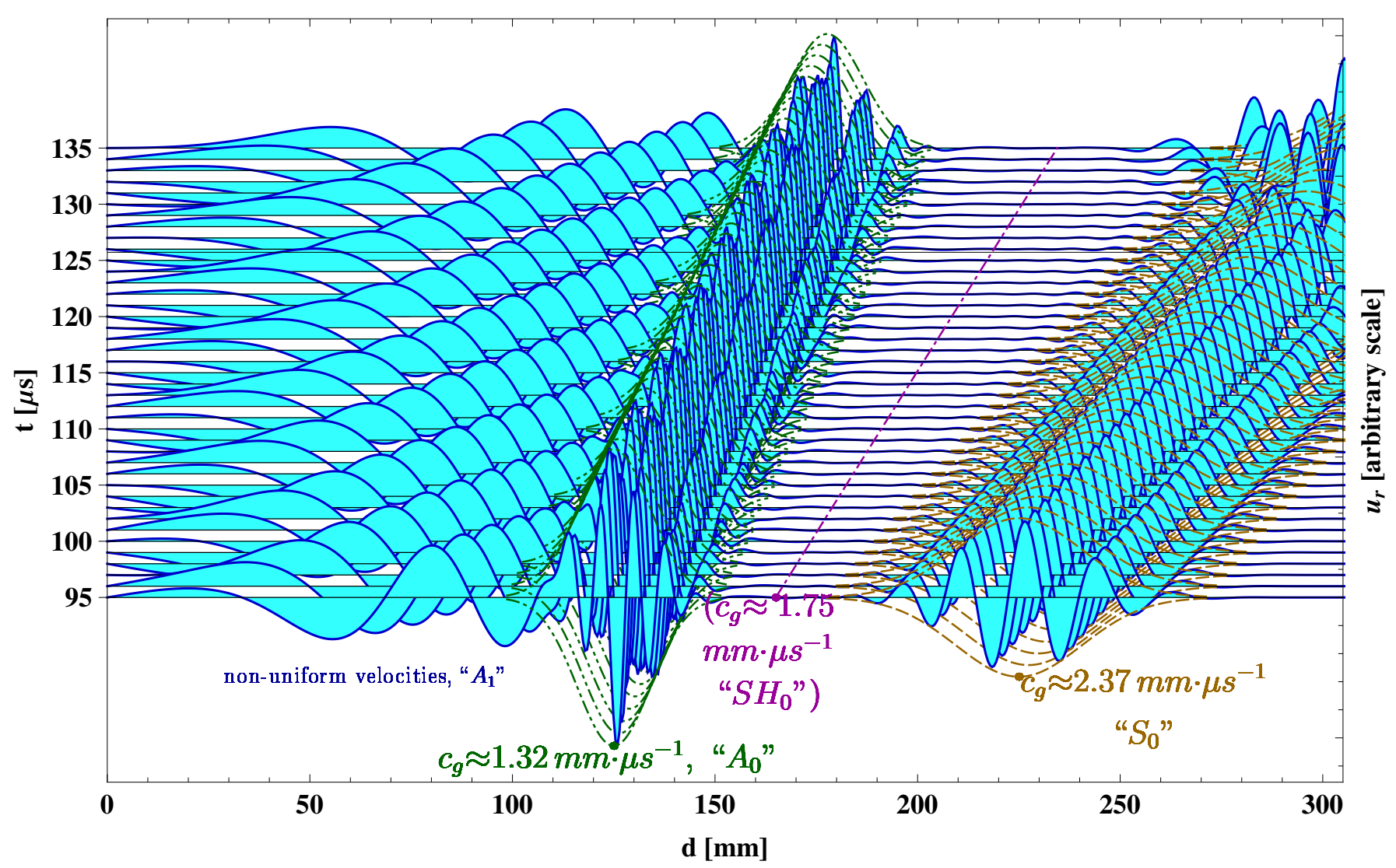

Figure 7: The radial displacement at $z=0$ and $x=0\left(\theta=90^{\circ}\right)$ in a carbon epoxy plate of thickness $3.6 \mathrm{~mm}$ excited by a 5-cycle pulse of frequency $150 \mathrm{kHz}$ at $z=0$, with respect to distance $d=y$ from the source and to time $t$.

(a) Radial displacement

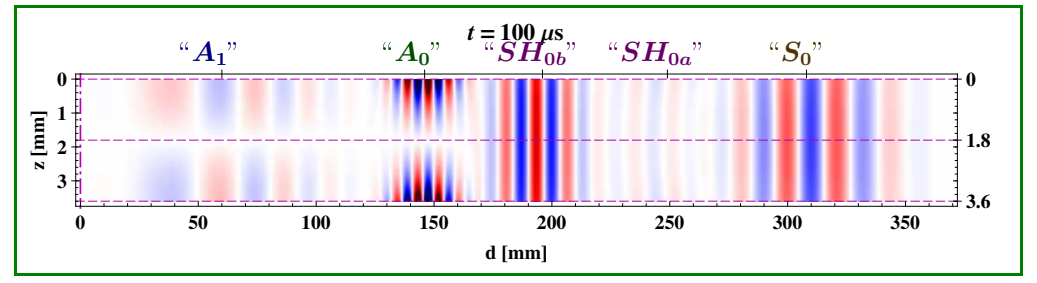

(b) Angular displacement

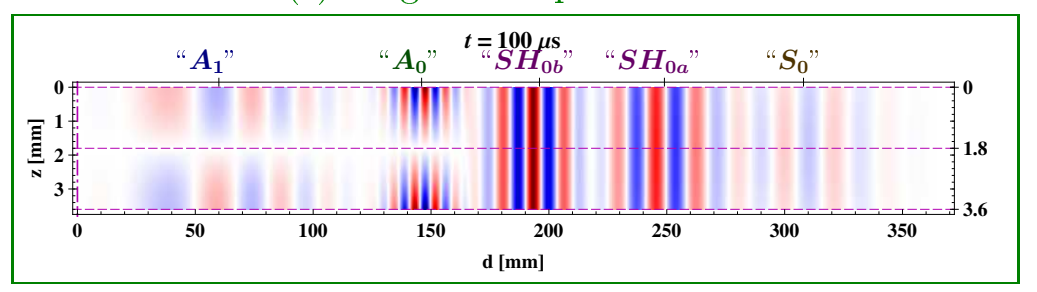

(c) Vertical displacement

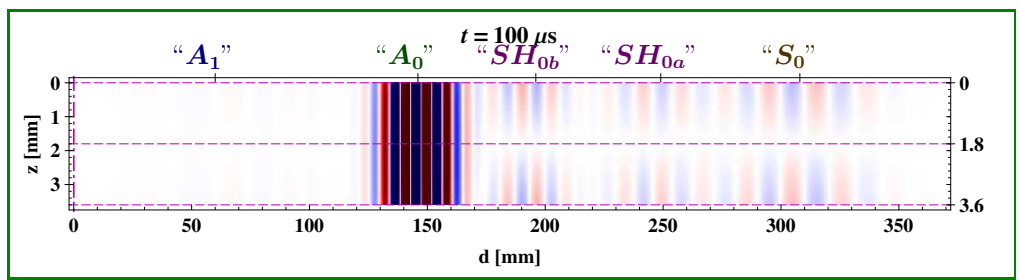

Figure 8: The displacements on section $\theta=43^{\circ}$ at $t=100 \mu \mathrm{s}$ in a carbon epoxy plate of thickness $3.6 \mathrm{~mm}$ excited by a 5 -cycle pulse of frequency $150 k H z$ at $z=0 . d$ is the distance from the source. 


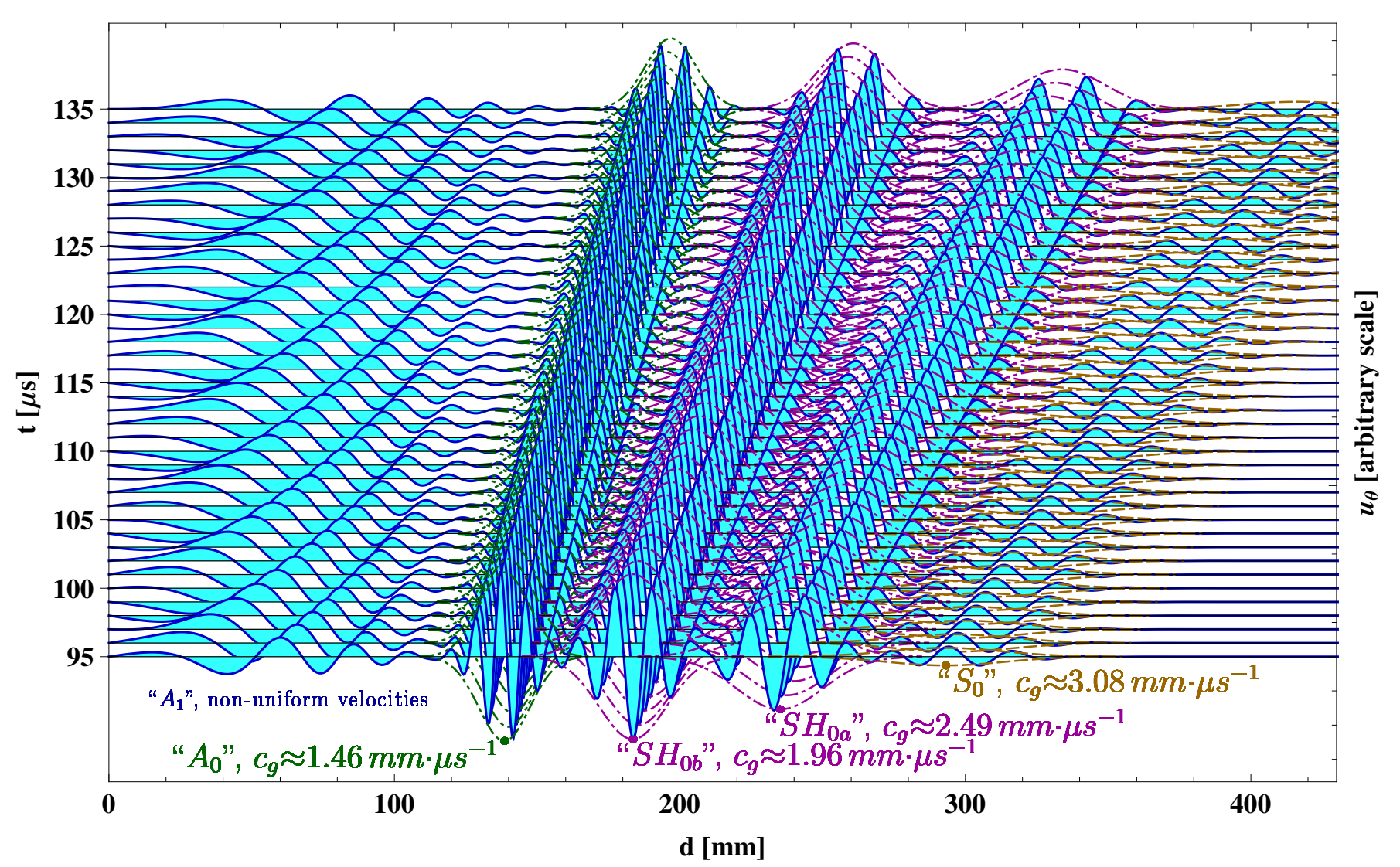

Figure 9: The angular displacement at $z=0$ in the direction $\theta=43^{\circ}$ in a carbon epoxy plate of thickness $3.6 \mathrm{~mm}$ excited by a 5-cycle pulse of frequency $150 \mathrm{kHz}$ at $z=0$, with respect to distance $d$ from the source and to time $t$.

\subsection{A unidirectional monolayer carbon fiber/epoxy plate glued to a steel plate}

As a final example, some results are presented for a plate made up of three layers. The upper layer is a monolayer made of the carbon epoxy composite plate analyzed in 5.1. The thickness is the same, i.e. $3.6 \mathrm{~mm}$, but the orientation of the plate is such that the crystallographic axis direction $\sharp 1$, the direction of the fibers, makes an angle of $\theta=45^{\circ}$ with the $x$-axis. For this kind of composite material, the higher velocities are in the fiber direction, then the farthest contributions, for a fixed time, are in this direction as well. Doing this rotation thus means that the 2D FFT sampling corresponds much better to the large extension of the field in this direction, as can be seen in Fig. 11, Direction $\sharp 3$ is still the $z$-axis.

The lower layer is a $1.0 \mathrm{~mm}$ thick steel plate of density $\rho=7900 \mathrm{~kg} \cdot \mathrm{m}^{-3}$. It is assumed to be isotropic. The stiffnesses are $c_{11}=280.0$ and $c_{44}=80.0[\mathrm{GPa}]$. These two layers are perfectly stuck together. The epoxy adhesive bond is $0.30 \mathrm{~mm}$ thick and assumed to be isotropic ("3M Scotch-Weld EC-9323 B/A Two Part Structural Adhesive" is considered here). Its mechanical properties are (from manufacturer's data in [22]): $\rho=1140 \mathrm{~kg} \cdot \mathrm{m}^{-3}$, $c_{11}=4.42$ and $c_{44}=1.07[\mathrm{GPa}]$.

The force field applied to the plane $z=0$ is oriented only in the $z$-direction with a spatial distribution in the interface plane given by an axisymmetric Gaussian of $17.0 \mathrm{~mm}$ in width. The signal is a 5 -cycle pulse of $100 \mathrm{kHz}$ frequency ( $c f$. Appendix B).

The radial displacement field calculated on the upper surface, for the fixed time $t=250 \mu s$, is plotted in Fig. 11 . The complexity of the response is clearly visible. First, let us note the accuracy of the numerical calculations. There is no numerical noise although the side of the square area of calculation is about 100 times the greatest wavelength. The same accuracy is obtained for a greater number of layers. As an example, numerical calculation has been carried out for a 25-layer composite with the same performances. From a physical point of view, the cusp can be perceived as well as many mode interferences. Analyzing the field in two cross-sections makes 


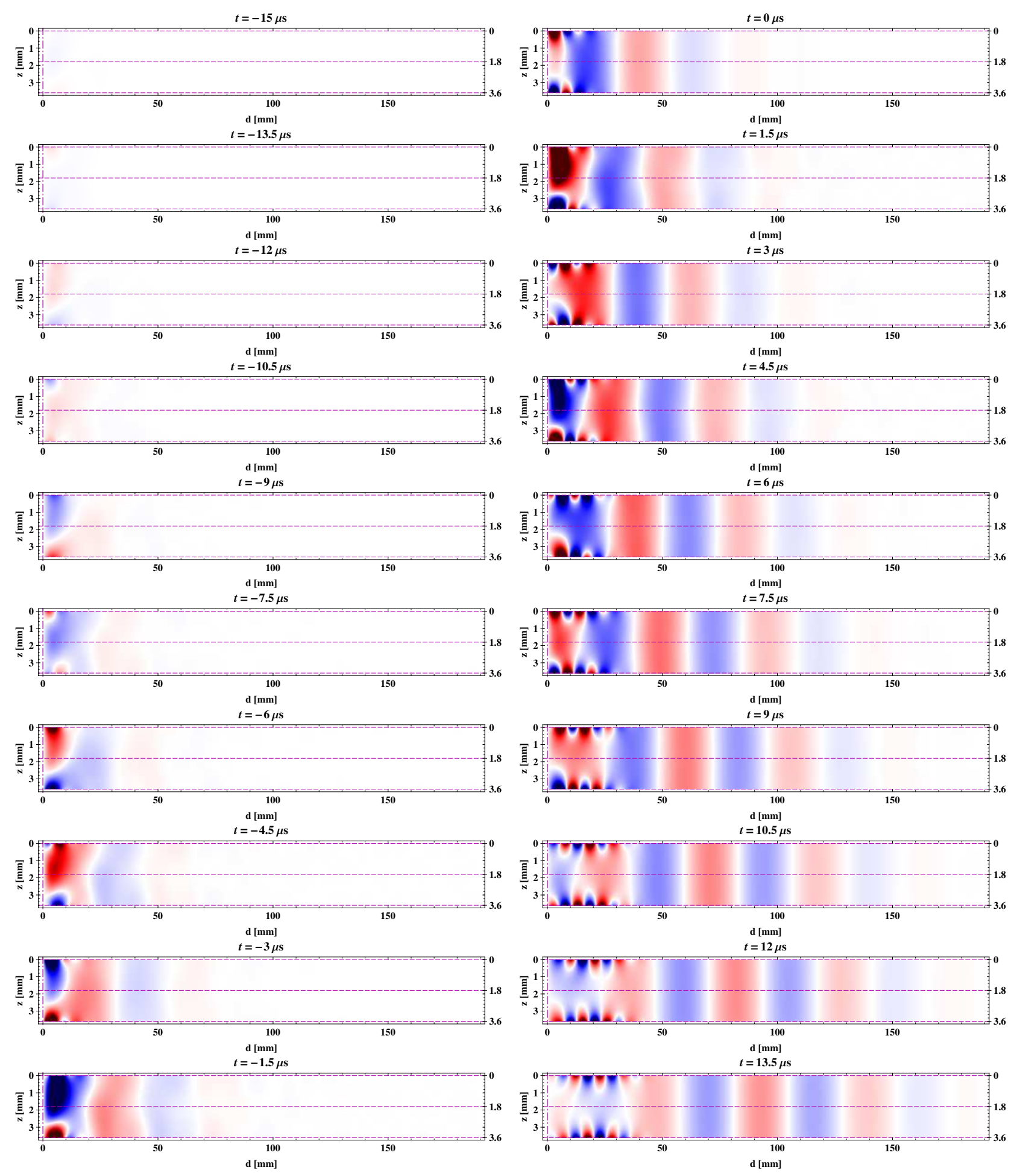

Figure 10: The radial displacement $u_{r}$ in the cross-section $x=0$ of a carbon epoxy plate of thickness $3.6 \mathrm{~mm}$ excited by a 5 -cycle pulse of frequency $150 \mathrm{kHz}$ at $z=0$, during the first microseconds.

interpretation of this response easier. In Figs. 12 and 13, the field is plotted versus the distance $d$ from the source center and the vertical position $z$ for the angles of $\theta=60^{\circ}$ and $\theta=90^{\circ}$, respectively.

Like the field in Fig. 8, the mode building, the existence of multiple rays due to the cusp and the dispersive behavior of one mode are clearly observed. Of course, in this case, owing to the inhomogeneity along the $z$-direction, the shape is much more complicated, although the field is periodic (or pseudo-periodic for the dispersive mode) along the ray directions. This periodicity depends directly on the projection on the ray direction of the wave-vector associated to the Lamb Wave. From that point of view, a generalized Lamb mode can be identified when this periodicity (or pseudo periodicity) exists. This is clearly the case in Fig. 13 for the 
angle $\theta=90^{\circ}$. In contrast, for the cross-section characterized by the angle $\theta=60^{\circ}$ (Fig. (12), no mode is distinctly identified between the distances 400 and $900 \mathrm{~mm}$, particularly when looking at the radial displacement. In fact, this reveals interferences between modes.

Generally, the energy of waves inside the plate is localized at different depths, either in agreement with the mode shapes ("pure" mode) or resulting from interferences between several modes.

(a) Radial Displacement at the upper face

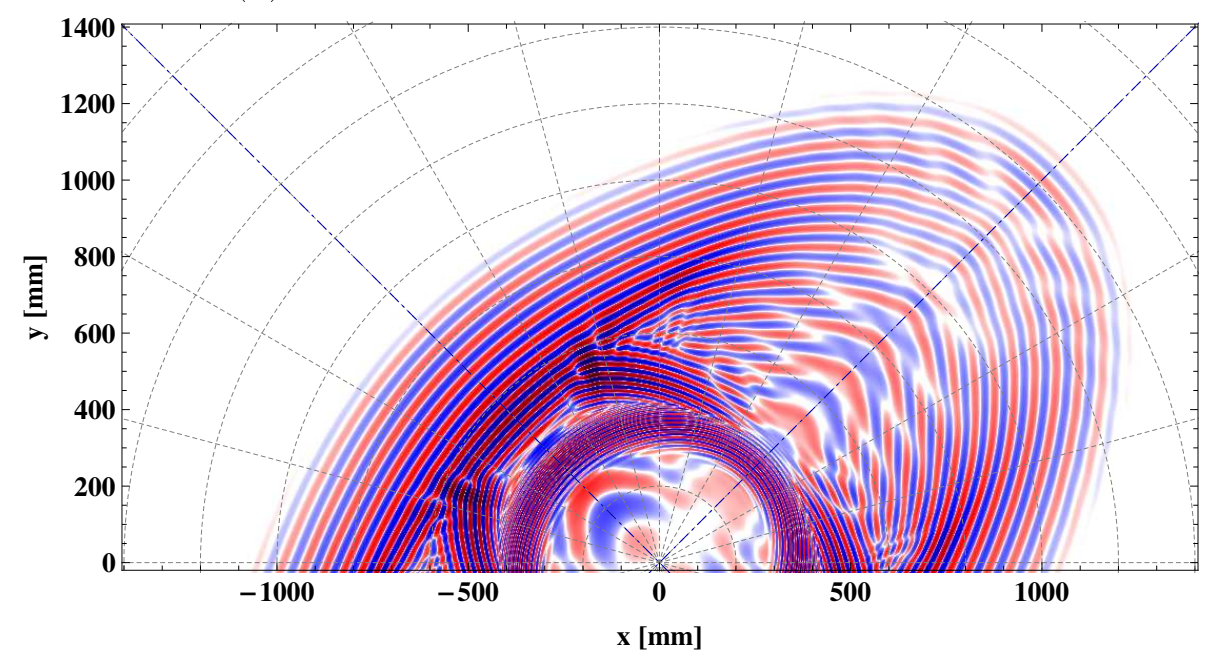

(b) Legend

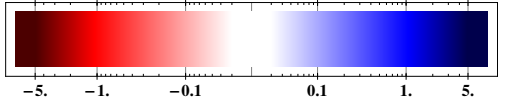

(arbitrary scale)

Figure 11: Response (radial displacement) at $z=0$ and $t=250 \mu \mathrm{s}$ of a multilayered plate, made of a monolayer composite plate and a steel plate glued together, to a 5 -cycle pulse of frequency $100 \mathrm{kHz}$ at $z=0$.

Radial displacement $u_{r}$

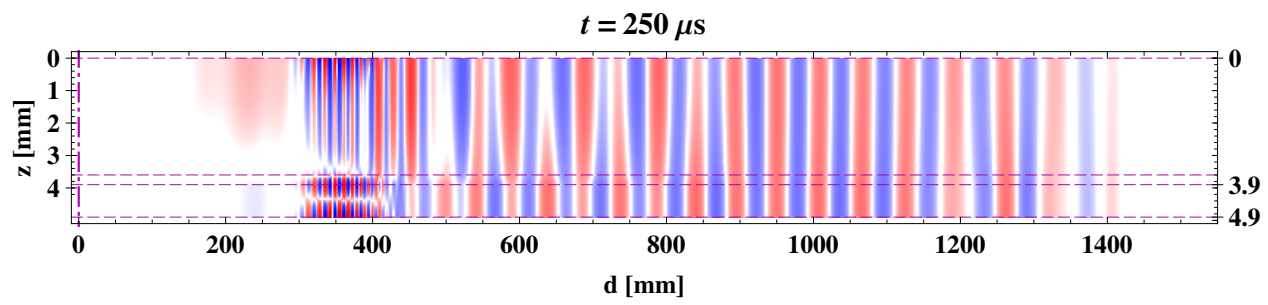

Angular displacement $u_{\theta}$

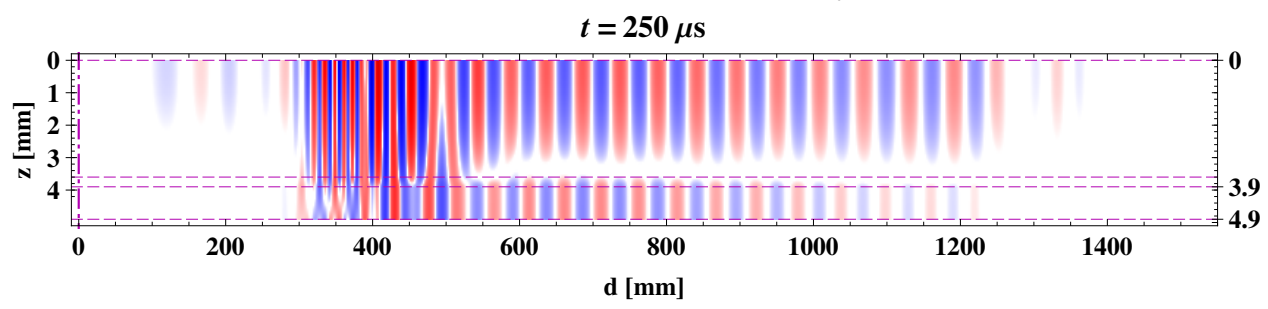

Vertical displacement $u_{z}$

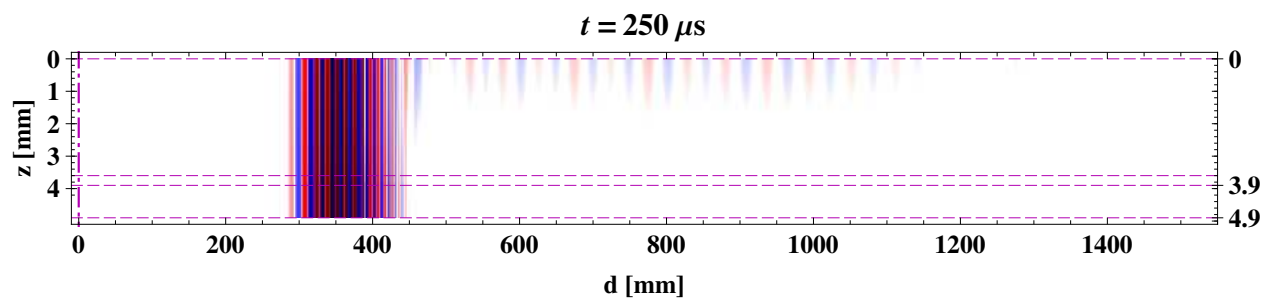

Figure 12: Response on section $\theta=60^{\circ}$ at $t=250 \mu \mathrm{s}$ of a multilayered plate, made of a monolayer composite plate and a steel plate glued together, to a 5 -cycle pulse of frequency $100 \mathrm{kHz}$ at $z=0$. 
Radial displacement $u_{r}$

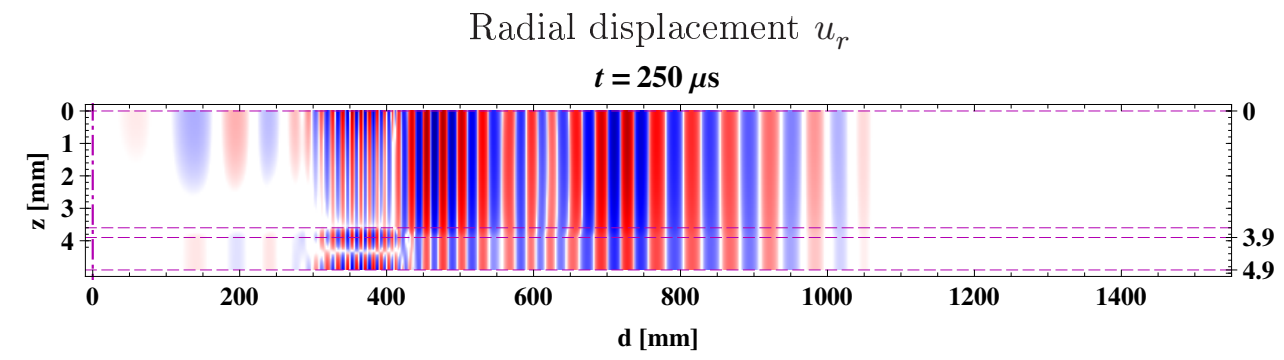

Angular displacement $u_{\theta}$

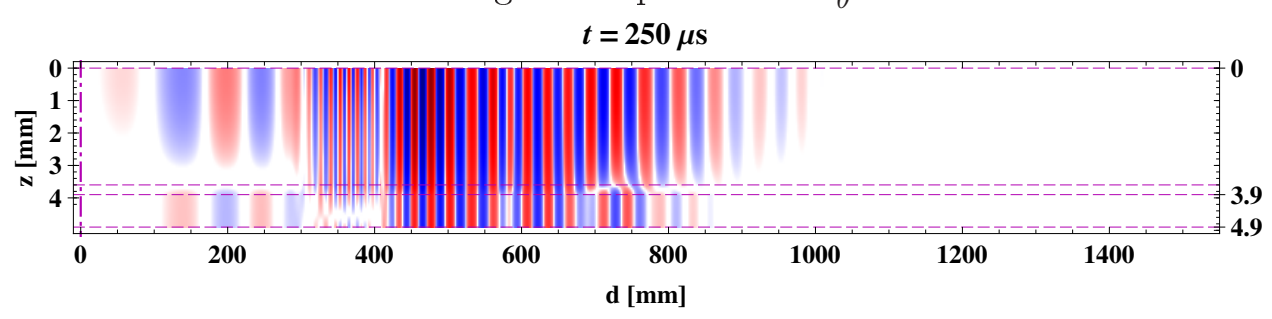

Vertical displacement $u_{z}$ $t=250 \mu \mathrm{s}$

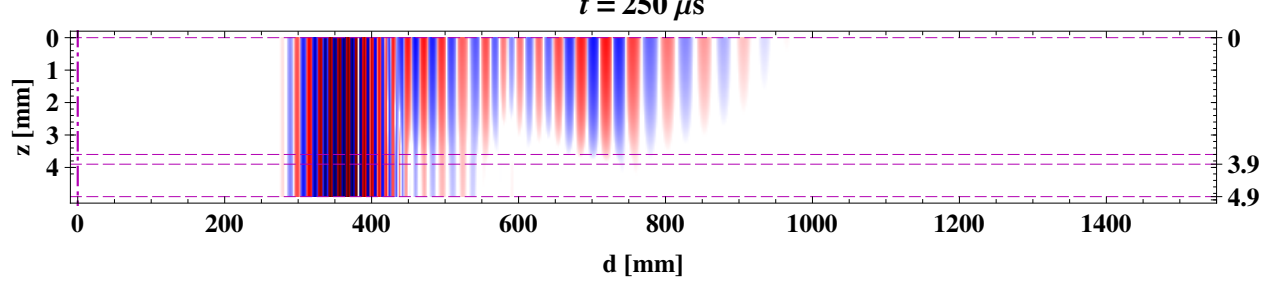

Figure 13: Response on section $\theta=90^{\circ}$ at $t=250 \mu \mathrm{s}$ of a multilayered plate, made of a monolayer composite plate and a steel plate glued together, to a 5 -cycle pulse of frequency $100 \mathrm{kHz}$ at $z=0$.

\section{Conclusion and future prospects}

The method that has been developed in this work, based on time-domain analysis, permits to calculate ultrasonic source diffractions in multilayered composites. This method is a useful tool which complements the most commonly used technique based on generalized Lamb wave decomposition, for which precise calculations can be made for long propagation distances, in a relatively short time. However, the most interesting feature of the modal decomposition in the time-domain is the fact that it is easier to calculate the acoustic response in the near field or under the source.

These calculations will be used to develop the topological imaging of defects hidden deep in sub-source positions in multilayered structures. The topological imaging method is based on interactions between two calculated fields, derived from the so-called direct and adjoint problems [23, 24, 25]. As these two fields are the results of wave propagation in a defectless medium, the present calculations will be highly suitable, especially if the defects are far from the source in the direction normal to the surface. This work, which also extends to cover unidirectional waveguides and immersed/embedded plates, is currently in progress. 


\section{References}

[1] B. L. N. Kennett, Seismic Wave Propagation in Stratified Media, Cambridge University Press, 1987.

[2] J. H. M. T. Van Der Hijden, Propagation of Transient Elastic Waves in Stratified Anisotropic Media (North-Holland Series in Applied Mathematics and Mechanics), Elsevier Science Ltd, 1987.

[3] A. Velichko, P. D. Wilcox, Modeling the excitation of guided waves in generally anisotropic multilayered media, J. Acoust. Soc. Am. 121 (1) (2007) 60-69. doi:10.1121/1.2390674.

[4] A. Karmazin, E. Kirillova, W. Seemann, P. Syromyatnikov, Investigation of Lamb elastic waves in anisotropic multilayered composites applying the Green's matrix, Ultrasonics 51 (1) (2011) 17-28. doi:10.1016/j.ultras.2010.05.003.

[5] M. J. S. Lowe, Matrix techniques for modeling ultrasonic waves in multilayered media, IEEE Trans. Ultrason. Ferroelectr. Freq. Control 42 (4) (1995) 525-542. doi:10.1109/58.393096.

[6] B. Hosten, M. Castaings, Surface impedance matrices to model the propagation in multilayered media, Ultrasonics 41 (7) (2003) 501-507. doi:10.1016/S0041-624X(03)00167-7.

[7] J.-M. Mencik, M. Ichchou, Multi-mode propagation and diffusion in structures through finite elements, Eur. J. Mech. A/Solids 24 (5) (2005) 877-898. doi:10.1016/j.euromechso1.2005.05.004.

[8] L. Taupin, A. Lhémery, V. Baronian, A.-S. Bonnet-BenDhia, B. Petitjean, Hybrid SAFE/FE model for the scattering of guided waves in a stiffened multi-layered anisotropic plate, in: Rev. Prog. QNDE, Vol. 31, D.O. Thompson and D.E. Chimenti, 2012.

[9] L. Taupin, A. Lhémery, V. Baronian, A.-S. Bonnet-BenDhia, Scattering of obliquely incident guided waves by a stiffener bonded to a plate, J. Phys.: Conf. Ser 353 (2012) 012011. doi:10.1088/1742-6596/353/1/012011.

[10] E. Kausel, Thin-Layer Method: formulation in the time domain, Int. J. Numer. Methods Eng. 37 (1994) 927-941.

[11] J. Park, E. Kausel, Response of layered half-space obtained directly in the time domain, Part I: SH sources, Bull. Seism. Soc. Am. 96 (2006) 1795-1809.

[12] F. J. Fedorov, Theory of elastic waves in crystals, Plenum Press / Kluwer Academic Publishers, New York, 1968.

[13] B. A. Auld, Acoustic Fields and Waves in Solids, 2nd Edition, Krieger Pub Co, 1990.

[14] M. J. P. Musgrave, Crystal acoustics, 2nd Edition, Acoust. Soc. Am., AIP, 2003.

[15] E. Ducasse, M. Deschamps, A nonstandard wave decomposition to ensure the convergence of Debye series for modeling wave propagation in an immersed anisotropic elastic plate, Wave Motion 49 (2012) 745-764.

[16] R. L. Weaver, W. Sachse, K. Y. Kim, Transient elastic waves in a transversely isotropic plate, J. Appl. Mech. 63 (2) (1996) 337-346.

[17] M. Géradin, D. Rixen, Mechanical Vibrations: Theory and Applications to Structural Dynamics, John Wiley \& Sons Ltd, 1997.

[18] N. Sloane, A. Wyner (Eds.), Claude Elwood Shannon: Collected Papers, IEEE Publications, 1993.

[19] J. W. Cooley, J. W. Tukey, An algorithm for the machine calculation of complex Fourier series, Math. Computation 19 (1965) 297-301. 
[20] G. Neau, Lamb waves in anisotropic viscoelastic plates: study of the wave fronts and attenuation, Ph.D. thesis, Bordeaux I University (2003).

[21] M. J. S. Lowe, G. Neau, M. Deschamps, Properties of guided waves in composite plates, and implications for NDE, AIP Conference Proceedings 700 (1) (2004) 214-221. doi:10.1063/1.1711627.

[22] [link].

URL http://multimedia.3m.com/mws/mediawebserver?mwsId=22222IiNZ6uG_LHhb40JaL7LtJiEt6iNWJg2

[23] M. Bonnet, B. B. Guzina, Sounding of finite solid bodies by way of topological derivative, International Journal for Numerical Methods in Engineering 61 (13) (2004) 2344-2373. doi:10.1002/nme.1153.

[24] N. Dominguez, V. Gibiat, Non-destructive imaging using the time domain topological energy method, Ultrasonics 50 (3) (2010) 367-372. doi:10.1016/j.ultras.2009.08.014.

[25] S. Rodriguez, M. Deschamps, M. Castaings, E. Ducasse, Guided wave topological imaging of isotropic plates, Ultrasonics 54 (7) (2014) 1880-1890. doi:10.1016/j.ultras.2013.10.001.

URL http://www.sciencedirect.com/science/article/pii/S0041624X13002862

[26] F. W. J. Olver, D. W. Lozier, R. F. Boisvert, C. W. Clark (Eds.), NIST Handbook of Mathematical Functions, Cambridge University Press, New York, 2010, print companion to [27].

[27] NIST Digital Library of Mathematical Functions, http://dlmf.nist.gov/, Release 1.0.5 of 2012-10-01, online companion to [26].

URL http://dlmf.nist.gov/

[28] P. A. Raviart, J. M. Thomas, Introduction à l'analyse numérique des équations aux dérivées partielles, Masson, Paris, 1983.

[29] H. Brezis, Functional Analysis, Sobolev Spaces and Partial Differential Equations, Universitext, Springer, New York, 2010. doi:10.1007/978-0-387-70914-7.

\section{Appendix A Spectral analysis of the $\mathbb{A}$ form}

\section{A.1 Theoretical background: density of the elastic potential energy}

The density of the potential energy of an elastically deformed body defined by (e.g., [12]):

$$
e_{p}=\frac{1}{2}\left(\partial_{x} \underline{\mathbf{u}} \cdot \underline{\boldsymbol{\sigma}}_{x}+\partial_{y} \underline{\mathbf{u}} \cdot \underline{\boldsymbol{\sigma}}_{y}+\partial_{z} \underline{\mathbf{u}} \cdot \underline{\boldsymbol{\sigma}}_{z}\right)
$$

is strictly positive for any non-zero deformation. A zero value of the density of the elastic potential energy $e_{p}$ corresponds to a rigid-body motion (translation and rotation).

The expression (A.1) becomes, by applying Hooke's Law (2):

$$
e_{p}=\frac{1}{2}\left[\begin{array}{c}
\partial_{x} \underline{\mathbf{u}} \\
\partial_{y} \underline{\mathbf{u}} \\
\partial_{z} \underline{\mathbf{u}}
\end{array}\right]^{\mathrm{T}}\left[\begin{array}{ccc}
(\mathbf{l} \diamond \mathbf{l}) & (\mathbf{l} \diamond \mathbf{m}) & (\mathbf{l} \diamond \mathbf{n}) \\
(\mathbf{m} \diamond \mathbf{l}) & (\mathbf{m} \diamond \mathbf{m}) & (\mathbf{m} \diamond \mathbf{n}) \\
(\mathbf{n} \diamond \mathbf{l}) & (\mathbf{n} \diamond \mathbf{m}) & (\mathbf{n} \diamond \mathbf{n})
\end{array}\right]\left[\begin{array}{c}
\partial_{x} \underline{\mathbf{u}} \\
\partial_{y} \underline{\mathbf{u}} \\
\partial_{z} \underline{\mathbf{u}}
\end{array}\right],
$$

where the unit vectors $\mathbf{l}, \mathbf{m}, \mathbf{n}$ are $\mathbf{n}_{x}, \mathbf{n}_{y}, \mathbf{n}_{z}$, respectively. Consequently, the nine-by-nine symmetric matrix in (A.2) is positive. 
In the 1D case where the displacement depends only on the $z$-coordinate, $e_{p}=\frac{1}{2} \partial_{z} \underline{\mathbf{u}}^{\mathbf{T}}(\mathbf{n} \diamond \mathbf{n}) \partial_{z} \underline{\mathbf{u}}$. Necessarily, the symmetric matrix $(\mathbf{n} \diamond \mathbf{n})$ is positively defined because rigid-body motions are only translations, for which $\partial_{z} \underline{\mathbf{u}}$ is zero.

In the $2 \mathrm{D}$ case where the displacement depends only on the $x$ and $z$ coordinates, the energy density becomes:

$$
e_{p}=\frac{1}{2}\left[\begin{array}{l}
\partial_{x} \underline{\mathbf{u}} \\
\partial_{z} \underline{\mathbf{u}}
\end{array}\right]^{\mathrm{T}} \mathcal{S}\left[\begin{array}{l}
\partial_{x} \underline{\mathbf{u}} \\
\partial_{z} \underline{\underline{u}}
\end{array}\right], \text { where } \mathcal{S}=\left[\begin{array}{ll}
(\mathbf{l} \diamond \mathbf{l}) & (\mathbf{l} \diamond \mathbf{n}) \\
(\mathbf{n} \diamond \mathbf{l}) & (\mathbf{n} \diamond \mathbf{n})
\end{array}\right] .
$$

In this second case the symmetric matrix $\mathcal{S}$ is positive but not defined because rigid-body motions include rotations in the $x-z$ plane, for which the spatial derivative of the displacement are non-zero. Indeed, because $(\mathbf{a} \diamond \mathbf{b}) \mathbf{c}=(\mathbf{a} \diamond \mathbf{c}) \mathbf{b}[15]$, it is obvious that $\mathcal{S}\left[\begin{array}{c}\mathbf{n} \\ -\mathbf{l}\end{array}\right]$ is the zero vector: $e_{p}=0$ if and only if $\partial_{x} \underline{u}_{x}, \partial_{x} \underline{u}_{y}, \partial_{z} \underline{u}_{y}, \partial_{z} \underline{u}_{z}$, and $\left(\partial_{x} \underline{u}_{z}+\partial_{z} \underline{u}_{x}\right)$ are zero.

\section{A.2 Definition of the $\mathcal{T}$ operator}

With the notation $\left.\Omega=\bigcup_{\beta=1}^{m}\right] z_{\beta-1}, z_{\beta}[=] 0, h \llbracket\left\{\left\{z_{1}, \cdots, z_{m-1}\right\}\right.$ ( $\Omega$ is an open bounded subset of $\mathbb{R}$ ), let us consider the following differential operator $\mathcal{T}$ involved in Eq. (8) and defined by:

$$
\forall z \in \Omega,(\mathcal{T} \boldsymbol{\Phi})(z)=\rho(z)^{-1}\left[-(\mathbf{n} \diamond \mathbf{n}) \boldsymbol{\Phi}^{\prime \prime}(z)+\dot{\mathrm{i}}[(\mathbf{n} \diamond \mathbf{k})+(\mathbf{k} \diamond \mathbf{n})] \boldsymbol{\Phi}^{\prime}(z)+(\mathbf{k} \diamond \mathbf{k}) \boldsymbol{\Phi}(z)\right]
$$

The $\mathcal{T}$ operator acts on any vector field $\boldsymbol{\Phi}$ satisfying the following conditions:

1. $\boldsymbol{\Phi}, \boldsymbol{\Phi}^{\prime}$ and $\boldsymbol{\Phi}^{\prime \prime}$ are defined on each interval $] z_{\beta-1}, z_{\beta}[$;

2. $\boldsymbol{\Phi}(0)=\boldsymbol{\Phi}\left(0^{+}\right), \boldsymbol{\Phi}^{\prime}(0)=\boldsymbol{\Phi}^{\prime}\left(0^{+}\right), \boldsymbol{\Phi}(h)=\boldsymbol{\Phi}\left(h^{-}\right), \boldsymbol{\Phi}^{\prime}(h)=\boldsymbol{\Phi}^{\prime}\left(h^{-}\right)$exist and:

- $(\mathbf{n} \stackrel{1}{\diamond} \mathbf{n}) \boldsymbol{\Phi}^{\prime}(0)-\dot{\mathrm{i}}(\mathbf{n} \stackrel{1}{\diamond} \mathbf{k}) \boldsymbol{\Phi}(0)=\mathbf{0}$ (upper side);

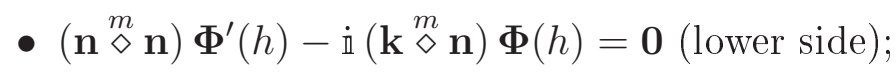

3. $\boldsymbol{\Phi}^{\prime \prime} \in \mathcal{L}^{2}([0, h])$, i.e. $\int_{0}^{h} \boldsymbol{\Phi}^{\prime \prime}(z)^{+} \boldsymbol{\Phi}^{\prime \prime}(z) \rho(z) d z<+\infty$, where the superscript + denotes the transposition combined with the complex conjugation;

4. Continuity on each interface $\sharp \beta\left(z=z_{\beta}\right.$, see Fig. 2):

- $\boldsymbol{\Phi}\left(z_{\beta}^{-}\right)=\boldsymbol{\Phi}\left(z_{\beta}^{+}\right)$(displacement);

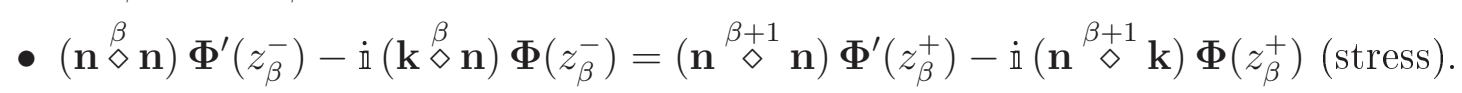

The set of such vector fields $\Phi$ is a functional space denoted by $\mathcal{V}$.

One can demonstrate that $\mathcal{V}$ is a subspace of the Sobolev space $\mathcal{H}^{2}(\Omega)$ and contains the test-function space $\mathcal{D}(\Omega)$, which is dense in $\mathcal{H}^{1}(\Omega)($ e.g, [28, Th. 1.2-3 (in French)] or [29]).

Furthermore, the Sobolev space $\mathcal{H}^{1}(\Omega)$ is dense in $\mathcal{L}^{2}([0, h])(e . g,[28])$, in which we consider the following scalar product of two functions:

$$
\forall \boldsymbol{\Phi}, \boldsymbol{\Psi} \in \mathcal{L}^{2}([0, h]),\langle\boldsymbol{\Phi}, \Psi\rangle=\int_{0}^{h} \boldsymbol{\Phi}(z)^{+} \boldsymbol{\Psi}(z) \rho(z) \mathrm{d} z=\sum_{\beta=1}^{m}\left[\rho_{\beta} \int_{z_{\beta-1}}^{z_{\beta}} \boldsymbol{\Phi}(z)^{+} \boldsymbol{\Psi}(z) \mathrm{d} z\right],
$$

and the canonical injection from $\mathcal{H}^{1}(\Omega)$ to $\mathcal{L}^{2}([0, h])$ is compact (e.g.,[28, Th. 1.5-2]) because $\Omega \subset[0, h]$ is bounded. 


\section{A.3 The hermitian form $\mathbb{A}$ associated to the $\mathcal{T}$ operator}

Let us consider the following sesquilinear form $\mathbb{A}$ :

$$
\forall \boldsymbol{\Phi}, \boldsymbol{\Psi} \in \mathcal{V}, \mathbb{A}(\boldsymbol{\Phi}, \Psi)=\langle\boldsymbol{\Phi}, \mathcal{T} \Psi\rangle=\sum_{\beta=1}^{m} \int_{z_{\beta-1}}^{z_{\beta}} \boldsymbol{\Phi}(z)^{+}(\mathcal{T} \boldsymbol{\Psi})(z) \mathrm{d} z
$$

After integration by parts on each interval $\left[z_{\beta-1}, z_{\beta}\right]$, we obtain:

$$
\begin{aligned}
& \mathbb{A}(\boldsymbol{\Phi}, \boldsymbol{\Psi})=\left\langle\boldsymbol{\Phi}^{\prime},(\mathbf{n} \diamond \mathbf{n}) \boldsymbol{\Psi}^{\prime}-\dot{\mathbb{i}}(\mathbf{n} \diamond \mathbf{k}) \boldsymbol{\Psi}\right\rangle+\dot{\mathbb{1}}\left\langle\boldsymbol{\Phi},(\mathbf{k} \diamond \mathbf{n}) \boldsymbol{\Psi}^{\prime}-\dot{\mathbb{i}}(\mathbf{k} \diamond \mathbf{k}) \Psi\right\rangle \\
& =\left\langle\Phi^{\prime},(\mathbf{n} \bullet \mathbf{n}) \Psi^{\prime}\right\rangle-\dot{\mathbb{1}}\left\langle\Phi^{\prime},(\mathbf{n} \bullet \mathbf{k}) \Psi\right\rangle+\dot{\mathbb{i}}\left\langle\boldsymbol{\Phi},(\mathbf{k} \diamond \mathbf{n}) \Psi^{\prime}\right\rangle+\langle\boldsymbol{\Phi},(\mathbf{k} \bullet \mathbf{k}) \Psi\rangle \\
& =\left\langle\Phi^{\prime},(\mathbf{n} \diamond \mathbf{n}) \Psi^{\prime}\right\rangle-\dot{\mathbb{i}}\left\langle\Phi^{\prime},(\mathbf{n} \diamond \mathbf{k}) \Psi\right\rangle+\dot{\mathbb{i}}\left\langle(\mathbf{n} \diamond \mathbf{k}) \boldsymbol{\Phi}, \Psi^{\prime}\right\rangle+\langle\boldsymbol{\Phi},(\mathbf{k} \diamond \mathbf{k}) \Psi\rangle \text {, }
\end{aligned}
$$

where $(\mathbf{a} \diamond \mathbf{b})$ is a three-by-three matrix such that $(\mathbf{a} \diamond \mathbf{b})=\rho_{\beta}^{-1}(\mathbf{a} \diamond \mathbf{b})$ on each layer $\sharp \beta$, and by using the fact that $(\mathbf{n} \diamond \mathbf{k})$ is equal to the transpose of the real-valued matrix $(\mathbf{k} \diamond \mathbf{n})$ (see [15]).

The definition (A.7) can be extended to functions $\boldsymbol{\Phi}$ and $\boldsymbol{\Psi}$ in the Sobolev space $\mathcal{H}^{1}(\Omega)$. The sesquilinear form $\mathrm{A}$ thus defined on $\mathcal{H}^{1}(\Omega)$ is hermitian, i.e. $\mathbb{A}(\boldsymbol{\Phi}, \boldsymbol{\Psi})=\mathbb{A}(\boldsymbol{\Psi}, \boldsymbol{\Phi})^{*}$, the superscript $*$ denoting the complex conjugation. Indeed, the matrices $(\mathbf{n} \bullet \mathbf{n})$ and $(\mathbf{k} \bullet \mathbf{k})$ are real-valued and symmetric [15].

\section{A.4 The hermitian form $A$ is positive}

If the wave-vector $\mathbf{k}$ is zero (1D case), $\mathrm{A}(\boldsymbol{\Phi}, \boldsymbol{\Phi})=\int_{0}^{h}\left[\boldsymbol{\Phi}^{\prime}(z)\right]^{+}(\mathbf{n} \diamond \mathbf{n}) \boldsymbol{\Phi}^{\prime}(z) \mathbb{d} z$.

$\mathrm{A}(\boldsymbol{\Phi}, \boldsymbol{\Phi})=0$ if and only if $\boldsymbol{\Phi}^{\prime}=\mathbf{0}$, i.e. the displacement field $\boldsymbol{\Phi}$ is uniform (translation).

If the wave-vector $\mathbf{k}$ is non-zero ( $2 \mathrm{D}$ case), without loss of generality, we can consider that the wave-vector $\mathbf{k}$ is parallel to the $x$ axis. Indeed, a rotation of the $x-y$ system leads to $\mathbf{k}=k \mathbf{l}$ and [see Eqs. (A.2) and (A.3)]: $\mathbb{A}(\boldsymbol{\Phi}, \boldsymbol{\Phi})=\int_{0}^{h}\left[\begin{array}{c}-\dot{\mathrm{i}} k \boldsymbol{\Phi}(z) \\ \boldsymbol{\Phi}^{\prime}(z)\end{array}\right]^{+} \mathcal{S}\left[\begin{array}{c}-\dot{\mathrm{i}} k \mathbf{\Phi}(z) \\ \boldsymbol{\Phi}^{\prime}(z)\end{array}\right] \mathbb{d} z \cdot \mathbb{A}(\boldsymbol{\Phi}, \boldsymbol{\Phi})=0$ if and only if $\boldsymbol{\Phi}=\mathbf{0}$.

Consequently, the hermitian form $\mathbf{A}$ is positive in any case, and positively defined if the wave-vector $\mathbf{k}$ is non-zero.

\section{A.5 Reduction of the hermitian form $\mathbb{A}$}

From the previous sections, it appears that the hermitian form $\mathbb{A}$ is coercitive (e.g., [28, Th. 6.2-1]), i.e. for all positive reals $\nu$, there is a positive real $\mu$ such that:

$$
\forall \boldsymbol{\Phi} \in \mathcal{H}^{1}(\Omega), \mathbb{A}(\boldsymbol{\Phi}, \boldsymbol{\Phi})+\nu\langle\Phi, \Phi\rangle \geqslant \mu\|\Phi\|_{\mathcal{H}^{1}(\Omega)}^{2}=\mu(\langle\boldsymbol{\Phi}, \boldsymbol{\Phi}\rangle+\langle\Phi, \boldsymbol{\Phi}\rangle)
$$

In conclusion, there are (e.g., [28, Th. 6.2-1]) both an increasing sequence $0 \leqslant \omega_{1}^{2} \leqslant \omega_{2}^{2} \leqslant \cdots \leqslant \omega_{n}^{2} \leqslant \cdots$ of positive eigenvalues which tends to infinity and an orthonormal Hilbert basis $\left\{\Phi_{n}\right\}_{n \in \mathbb{N}^{*}}$ of $\mathcal{L}^{2}(\Omega)$, i.e.

$$
\forall n, n^{\prime} \in \mathbb{N}^{*}, n \neq n^{\prime},\left\langle\Phi_{n}, \Phi_{n}\right\rangle=1 \text { and }\left\langle\Phi_{n}, \Phi_{n^{\prime}}\right\rangle=0
$$

and

$$
\forall \boldsymbol{\Psi} \in \mathcal{L}^{2}(\Omega), \boldsymbol{\Psi}=\sum_{n=1}^{+\infty}\left\langle\boldsymbol{\Phi}_{n}, \boldsymbol{\Psi}\right\rangle \boldsymbol{\Phi}_{n}
$$


such that $\Phi_{n} \in \mathcal{H}^{1}(\Omega)$ for all integers $n$ and:

$$
\forall \boldsymbol{\Psi} \in \mathcal{H}^{1}(\Omega), \mathbb{A}\left(\Phi_{n}, \boldsymbol{\Psi}\right)=\omega_{n}^{2}\left\langle\Phi_{n}, \boldsymbol{\Psi}\right\rangle
$$

Each pair $\left(\omega_{n}, \boldsymbol{\Phi}_{n}\right)$ defines an eigenmode: $\omega_{n}$ is its angular eigenfrequency and $\boldsymbol{\Phi}_{n}$ the mode shape.

Note that the mode shape $\boldsymbol{\Phi}_{n}$ is an element of the Sobolev space $\mathcal{H}^{1}(\Omega)$ but is not necessarily an element of the $\mathcal{V}$ space. Nevertheless, $\Phi_{n}$ can be approached as near as desired by an element of $\mathcal{V}\left(\mathcal{V}\right.$ is dense in $\left.\mathcal{H}^{1}(\Omega)\right)$

\section{Appendix B Gaussian and $n$-cycle pulses: definition and convolution by causal sine signals}

\section{B.1 Gaussian pulse of duration $d$}

Consider the Gaussian pulse $g_{d}$ of "duration" $d$ defined as follows:

Time-domain : $g_{d}(t)=\frac{5}{2 d} \exp \left(\frac{-25 \pi t^{2}}{4 d^{2}}\right) \Longleftrightarrow$ Frequency-domain : $\hat{g}_{d}(\omega)=\exp \left(\frac{-d^{2} \omega^{2}}{25 \pi}\right)$

such that $g_{d}$ tends to the Dirac delta function when the duration tends to zero, as drawn in Fig. B.1,

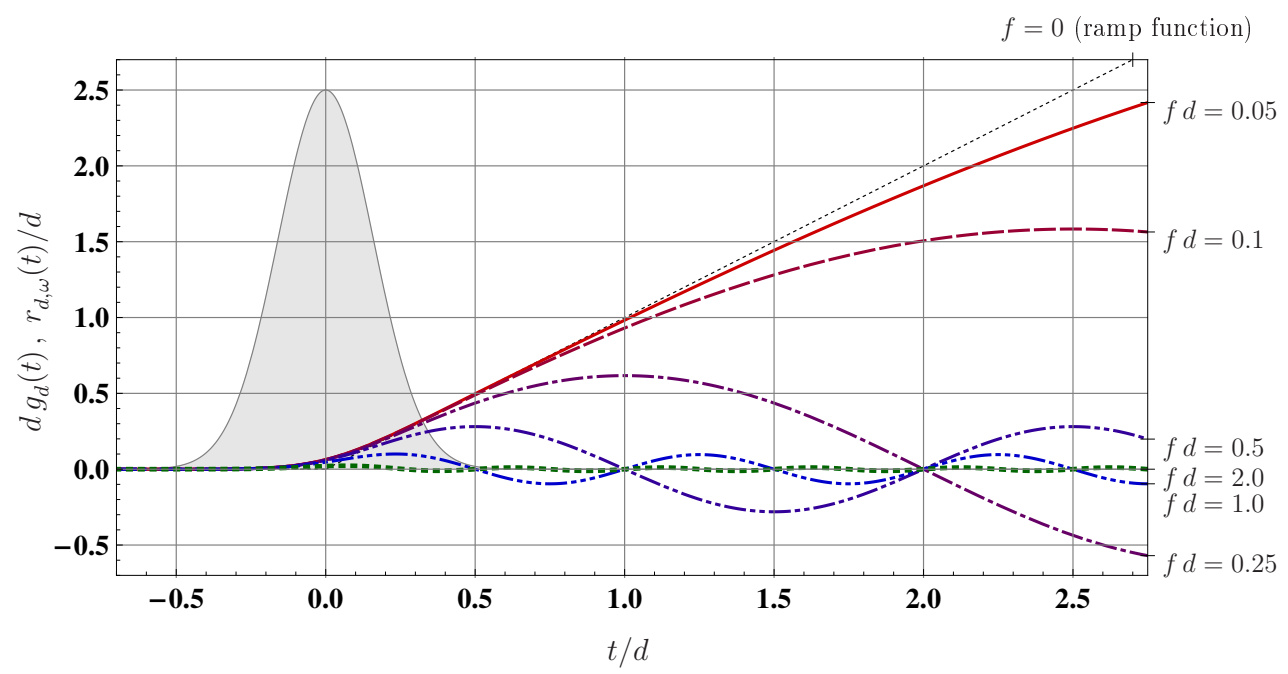

Figure B.1: The convolution of a Gaussian pulse (plain, thin) of duration $d$ by a causal sine signal of frequency $f$, for $f=0$ (dashed thin), $f d=0.05$ (plain), $f d=0.1$ (dashed), $f d=0.25(-\cdot), f d=0.5(-\cdot \cdot), f d=1(-\cdots), f d=2$ (dotted thick).

One can demonstrate that the convolution of the Gaussian pulse by the causal sine of angular frequency $\omega$ is $r_{d, \omega}$ given by:

$$
\begin{aligned}
r_{d, \omega}(t) & =\int_{-\infty}^{t} g_{d}(\tau) \frac{\sin [\omega(t-\tau)]}{\omega} \mathbb{d} \tau \\
& =\frac{1}{\omega} \exp \left(\frac{-d^{2} \omega^{2}}{25 \pi}\right)\left[\sin (\omega t)+\frac{1}{2} \mathcal{I} m\left\{\mathbb{e}^{\dot{\mathrm{i}} \omega t}\left[\operatorname{erf}\left(\frac{5 \sqrt{\pi}}{2 d} t+\dot{\mathrm{i}} \frac{\omega d}{5 \sqrt{\pi}}\right)-1\right]\right\}\right]
\end{aligned}
$$

where "erf" denotes the error function defined by: $\operatorname{erf}(z)=2 \sqrt{\pi}^{-1} \int_{0}^{z} \mathbb{e}^{-\zeta^{2}} \mathbb{d} \zeta($ e.g., [27, $\left.\S 7.2]\right)$. 
As expected, because $\operatorname{erf}(a+\dot{\mathrm{i}} b)$ tends to \pm 1 when $a$ tends to $\pm \infty$, we can observe that the convolution product is zero before the beginning of the pulse and it is $\frac{\sin (\omega t)}{\omega} \exp \left(\frac{-d^{2} \omega^{2}}{25 \pi}\right)=\frac{\hat{g}_{d}(\omega)}{\omega} \sin (\omega t)[\mathrm{Eq}$. (B.1)] after the end of the pulse (see Fig. B.1).

\section{B.2 $n$-cycle pulse of angular frequency $\nu$}

Consider the $n$-cycle pulse $p_{n, \nu}$ of angular frequency $\nu$ defined by:

$$
\overbrace{\text { Frequency-domain : } \hat{p}_{n, \nu}(\omega)=\frac{1}{2 \dot{\mathrm{i}}}\left\{\exp \left[\frac{-\pi^{2} n^{2}(\omega-\nu)^{2}}{18 \nu^{2}}\right]-\exp \left[\frac{-\pi^{2} n^{2}(\omega+\nu)^{2}}{18 \nu^{2}}\right]\right\}}^{\mathbb{1}} .
$$

This $n$-cycle pulse and its spectrum are drawn in Fig. B.2, for $n=2,3$ and 5 .

$(a)$

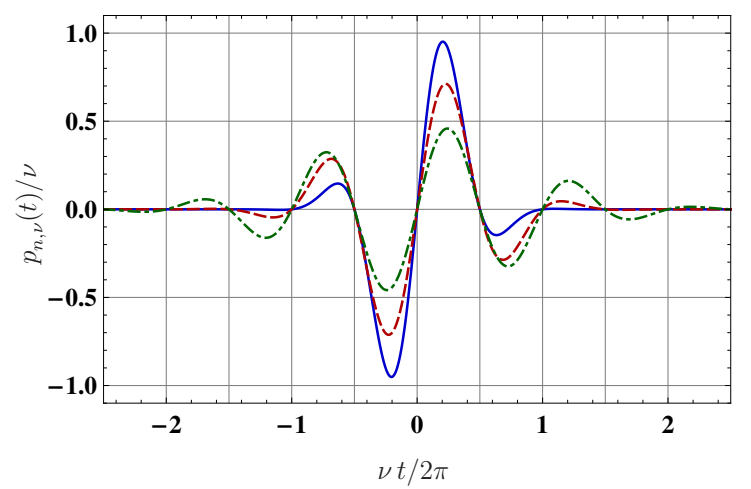

(b)

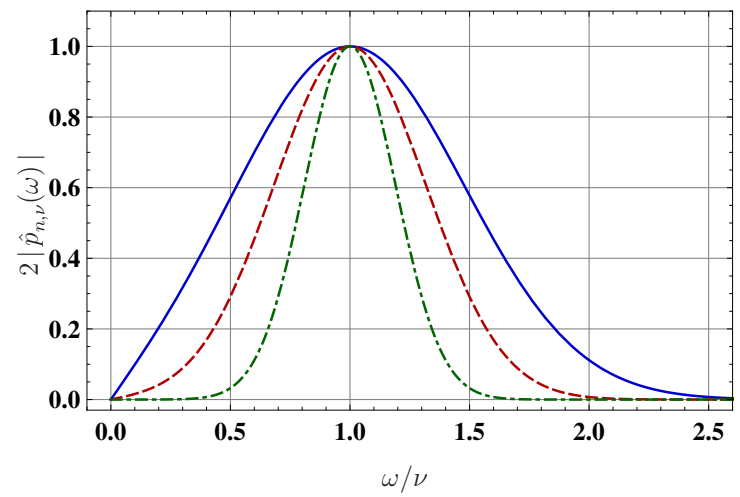

Figure B.2: $n$-cycle pulses of angular frequency $\nu,(a)$ in the time-domain and $(b)$ in the frequency-domain, where $n=2$ (plain), $n=3$ (dashed) and $n=5$ (dot-dashed).

The convolution of the $n$-cycle pulse by the causal sine of angular frequency $\omega$ is $r_{n, \nu, \omega}$ given by:

$$
\begin{aligned}
r_{n, \nu, \omega}(t)= & \int_{-\infty}^{t} p_{n, \nu}(\tau) \frac{\sin [\omega(t-\tau)]}{\omega} \mathbb{d} \tau \\
= & \frac{1}{4 \omega}\left\{\mathbb{e}^{-b_{+}^{2}}\left[\left(1+r_{+}(t)\right) \cos (\omega t)-i_{+}(t) \sin (\omega t)\right]\right. \\
& \left.-\mathbb{e}^{-b_{-}^{2}}\left[\left(1+r_{-}(t)\right) \cos (\omega t)+i_{-}(t) \sin (\omega t)\right]\right\},
\end{aligned}
$$

where:

$$
b_{ \pm}=\frac{n \pi(\nu \pm \omega)}{3 \sqrt{2} \nu}, c_{ \pm}(t)=\operatorname{erf}\left(\frac{3 \nu}{n \pi \sqrt{2}} t+\dot{\mathrm{i}} b_{ \pm}\right), r_{ \pm}(t)=\mathcal{R} e\left[c_{ \pm}(t)\right] \text { and } i_{ \pm}(t)=\mathcal{I} m\left[c_{ \pm}(t)\right] .
$$

As above, because $\operatorname{erf}(a+\dot{\mathbb{i}} b)$ tends to \pm 1 when $a$ tends to $\pm \infty$, we can observe that the convolution product is zero before the beginning of the pulse and it is $\frac{\cos (\omega t)}{\omega} \frac{\mathbb{e}^{-b_{+}^{2}-\mathbb{e}^{-b_{-}^{2}}}}{2}=\frac{-\left|\hat{p}_{n, \nu}(\omega)\right|}{\omega} \cos (\omega t)[$ Eq. (B.3) $)$ after the end of the pulse (see Fig. B.3). 


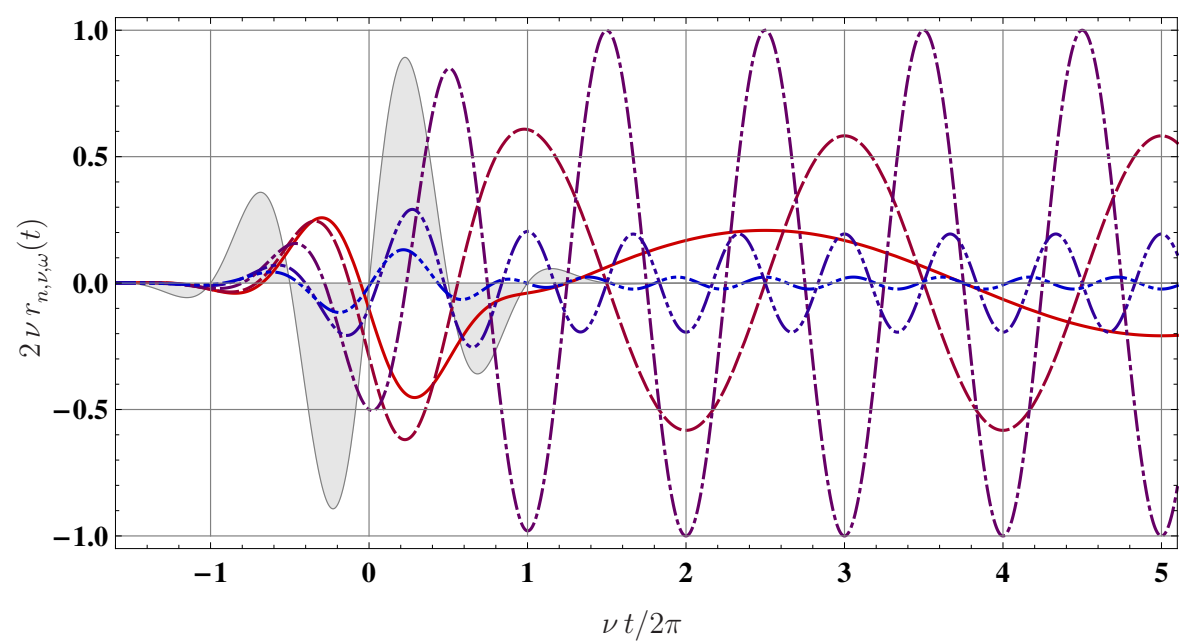

Figure B.3: The convolution of a 3-cycle pulse (plain, thin) by a causal sine signal of angular frequency $\omega, \omega=0.2 \nu$ (plain), $\omega=0.5 \nu$ (dashed), $\omega=\nu(-\cdot), \omega=1.5 \nu(-\cdot \cdot), \omega=1.8 \nu(-\cdots)$. 